\title{
Vegan-vegetarian low-protein supplemented diets in pregnant CKD patients: fifteen years of experience
}

Rossella Attini, Filomena Leone, Silvia Parisi, Federica Fassio, Irene Capizzi ${ }^{1}$, Valentina Loi ${ }^{4}$, Loredana Colla ${ }^{5}$ Maura Rossetti ${ }^{5}$, Martina Gerbino, Stefania Maxia ${ }^{4}$, Maria Grazia Alemanno, Fosca Minelli, Ettore Piccoli, Elisabetta Versino ${ }^{2,3}$, Marilisa Biolcati, Paolo Avagnina ${ }^{6,7}$, Antonello Pani ${ }^{4}$, Gianfranca Cabiddu ${ }^{4}$, Tullia Todros and Giorgina B. Piccoli, ${ }^{6 *}$

\begin{abstract}
Background: Pregnancy in women with advanced CKD becoming increasingly common. However, experience with low-protein diets in CKD patients in pregnancy is still limited.

Aim of this study is to review the results obtained over the last 15 years with moderately restricted low-protein diets in pregnant CKD women (combining: CKD stages 3-5, proteinuria: nephrotic at any time, or > =1 g/24 at start or referral; nephrotic in previous pregnancy). CKD patients on unrestricted diets were employed for comparison.

Methods: Study period: January, 2000 to September, 2015: 36 on-diet pregnancies (31 singleton deliveries, 3 twin deliveries, 1 pregnancy termination, 1 miscarriage); 47 controls (42 singleton deliveries, 5 miscarriages). The diet is basically vegan; since occasional milk and yoghurt are allowed, we defined it vegan-vegetarian; protein intake (0.6-0.8 g/Kg/day), keto-acid supplementation, protein-unrestricted meals (1-3/week) are prescribed according to CKD stage and nutritional status. Statistical analysis was performed as implemented on SPSS.
\end{abstract}

Results: Patients and controls were similar (p: ns) at baseline with regard to age (33 vs 33.5), referral week (7 vs 9), kidney function (CKD 3-5: $48.4 \%$ vs $64.3 \%$ ); prevalence of hypertension (51.6\% vs $40.5 \%$ ) and proteinuria >3 g/ $24 \mathrm{~h}$ (16.1\% vs $12.2 \%$ ). There were more diabetic nephropathies in on-diet patients (on diet: $31.0 \%$ vs controls 5. $3 \%$; p 0.007 (Fisher)) while lupus nephropathies were non-significantly higher in controls (on diet: $10.3 \%$ vs controls $23.7 \% ;$ p 0.28 (Fisher)). The incidence of preterm delivery was similar (<37 weeks: on-diet singletons 77. $4 \%$; controls: $71.4 \%$ ). The incidence of other adverse pregnancy related outcomes was non-significantly lower in on-diet patients (early preterm delivery: on diet: $32.3 \%$ vs controls $35.7 \%$; birth-weight $=<1.500$ g: on diet: $9.7 \%$ vs controls $23.8 \%)$. None of the singletons in the on-diet series died, while two perinatal deaths occurred among the controls $(p=0.505)$.

The incidence of small for gestational age (SGA <10th centile) and/or extremely preterm babies (<28th week) was significantly lower in singletons from on-diet mothers than in controls (on diet: $12.9 \%$ vs controls: $33.3 \%$; p: 0.04 (Fisher)).

(Continued on next page)

\footnotetext{
* Correspondence: gbpiccoli@yahoo.it

${ }^{6} \mathrm{SS}$ Nephrology, Department of Clinical and Biological Sciences, University of

Torino, Regione Gonzole 10, Orbassano, Torino 10100, Italy

${ }^{7}$ Nèphrologie, $\mathrm{CH}$ du Mans, Le Mans, France

Full list of author information is available at the end of the article
} 
(Continued from previous page)

Conclusion: Moderate protein restriction in the context of a vegan-vegetarian supplemented diet is confirmed as a safe option in the management of pregnant CKD patients.

Keywords: Low-protein diets, Supplemented diets, Pregnancy, CKD, Maternal-foetal outcomes, Small for gestational age baby, Preterm delivery

\section{Background}

When we prescribed a low-protein diet to the first pregnant patient with severe proteinuria and diabetic nephropathy (a case which gave us the opportunity to start a "joint venture" between Nephrology and Obstetrics), we did not foresee that fifteen years later our Unit would have followed-up a few hundred pregnancies, about $5 \%$ of which involved subjects on a protein-restricted diet [1-5]. We also did not foresee that several large studies would have challenged the "meat eaters" in favour of Mediterranean or vegetable-based diets, thus leading to reconsider the role of protein intake in the overall population, as well as in CKD [6-14]. We were mainly worried about the patient's increasing levels of proteinuria, and we did not know what else we could do besides keeping blood pressure under control, ordering bed rest (still a widely used procedure) and checking the baby's growth curve [1].

On the basis of the available data on hyper-filtration in CKD and on the effect of low-protein diets in reducing the "work load" on the remnant nephrons, we chose to start her on the diet that we considered the "best" one available in our hands, i.e. a low-protein, vegan, supplemented diet [15-18].

After our patient delivered a healthy male baby, adequate for gestational age, at the 30th gestational week, we started prescribing a low-protein, vegan-vegetarian diet, with a simplified qualitative schema, to other pregnant patients with severe kidney function impairment or relevant proteinuria [1]. Our first results, involving 12 pregnancies, were promising enough to double the number of patients in a few years [2, 4]. The subsequent analysis on 22 live-born singleton deliveries showed the almost paradoxical finding of better growth in children delivered by on-diet mothers as compared to children of CKD mothers on an unrestricted diet [4].

At the time of our first experiences, 1-1.2 $\mathrm{g}$ of proteins / Kg day was considered the "normal" protein intake, and the intake in pregnant women was often higher, thus making our diets conflicting with the common beliefs in pregnancy. However, interest in veganvegetarian diets grew over the following years, and they are now considered safe in all phases of life, including pregnancy and lactation, provided that vitamins and microelements were controlled and integrated when needed [19-31].
Meanwhile, we gradually integrated the recommendation that patients should avoid both excessive weight gain; this was carried out by shifting from a purely qualitative diet prescription to the present qualitativequantitative one [2, 4] (Appendix).

The main drawback of our previous studies was the difficulty of recruiting a homogeneous control group [2, 4]. Thus, the novelty of the present analysis, which is aimed at reviewing the results gathered over 15 years, is that the results of on-diet pregnancies are compared to a composite larger control group of pregnancies with similar clinical characteristics.

\section{Methods \\ Definitions and control policies}

CKD was defined and staged according to K-DOQI guidelines, whenever possible according to pre-conceptional data. Throughout pregnancy, GFR and proteinuria were assessed by 24-h urine collections, as specified more in detail elsewhere [5].

A newborn was defined as Small for Gestational Age (SGA) when birth weight was below the 5 th or below the 10th centile, according to the birth weight references that were used [32-34]. Due to the specific interest in this point, we employed both the older Italian Parazzini charts and the newer INeS (Italian Neonatal Study) charts, and analysed the two cut-points at the 5th and 10 th percentile $[33,34]$. Preterm delivery, early preterm delivery and extremely preterm delivery were defined as before 37, 34 and 28 completed weeks of gestational age, respectively [32].

Hypertension was defined as per the current guidelines; the antihypertensive treatment was mainly based upon a combination of alphamethyl-dopa and nifedipine, adding doxazosine, small doses of diuretics or clonidine only when absolutely needed. Treatment was adjusted at every clinical visit with a target of 120-130/60-70 mmHg [5].

The study was performed in two Italian settings: Torino and Cagliari. These are the two Centers with the greatest experience of management of CKD in pregnancy in Italy, that keep a conjoint database (TOCOS: Torino Cagliari Observational Study [5]). For the sake of this study, the cases were recruited in Torino, the controls were selected in both settings, as further specified. In both settings of care, the frequency of nephrological 
and obstetric visits, of blood and urine tests and of biometric and Doppler studies of uterine and umbilical arteries are tailored to the individual patient (visits: 1 week -1 month, biometry every 2-3 weeks in case of SGA babies or at risk for foetal growth restriction; Doppler assessment two-three times weekly in case of Doppler anomalies), in keeping with the Italian best practices in pregnant CKD patients $[35,36]$.

\section{The low-protein diet}

The low protein diet consisted in an adaptation of the low-protein vegan diet employed in our centre, itself a simplification of the original scheme by Barsotti and Giovannetti $[17,18]$.

Unlike the Barsotti and Giovannetti diets, our basic schemas are simplified: the food is chosen according to a qualitative approach (allowed-forbidden), not weighed, with a protein intake of $0.6 \mathrm{~g} / \mathrm{Kg} /$ day (ideal weight), and 1-3 free meals per week. To allow the patient to follow a vegan diet without the need to use legumes and cereals in each meal, we added a supplementation of alpha-keto analogues and aminoacids (Alpha-Kappa or Ketosteril according to the availability over time): 1 pill/10 Kg of ideal body weight $[37,38]$.

In an empirical attempt to balance the potential advantages of low-protein diets in CKD and the habit of increasing protein intake in pregnancy, we initially adjusted the diet from 0.6 to $0.6-0.8 \mathrm{~g} / \mathrm{Kg} /$ day of proteins, based on pre-conception weight, usually by increasing the protein intake from the first $(0.6 \mathrm{~g} / \mathrm{Kg} /$ day $)$ to the last trimester $(0.8 \mathrm{~g} / \mathrm{Kg} /$ day $)$. We also increased the amino and keto-acids supplementation from 1 pill each $10 \mathrm{Kg}$ to 1 pill each $8 \mathrm{Kg}$, and in patients with low body weight, even up to 1 pill each $5 \mathrm{Kg}$ in late pregnancy.

At the time of the first case, no report on these issues had been found or made available by the company; no report on safety concerns was available at that time or was found at the subsequent updates.

Since patients often missed milk and yoghurt in their diets, we allowed small quantities (100-150 mL per day) in selected cases, and changed the definition of "vegan" into "vegan-vegetarian". On the basis of the functional status, of the proteinuria levels and of the patients' needs and preferences, in keeping with the policy applied to non-pregnant patients, we allowed 1-3 unrestricted meals per week (without protein restriction but limited in unsaturated fats and short-chain sugars).

On the account of the lack of indications on salt restriction in pregnancy, we did not restrict salt; since salt intake cannot be controlled by the analysis of the $24 \mathrm{~h}$ excretion in pregnancy, due to the lack of referral standards, we limited our interventions to diet counselling in the cases with severe oedema or uncontrolled hypertension.
In addition to the biochemical tests (targeted at CKD), we progressively added iron status, $\mathrm{B} 12$, and $25-\mathrm{OH}$ vitamin D to the routine monthly tests; vitamins and iron supplements were employed on the basis of the biochemical results. Erythropoietin was used when needed, with a haemoglobin target of $10 \mathrm{~g} / \mathrm{dL}$ on account of the physiological haemodilution of pregnancy.

The most recent version of the diet is reported in the Appendix.

\section{Indications for the diet and selection of controls}

The main indications for the low-protein veganvegetarian diets in pregnancy were progressively broadened from the initial subjects with CKD stages 4-5 and/ or nephrotic syndrome to include pregnancy in patients already on a supplemented vegetarian diet; CKD stages $3 \mathrm{~b}$ or 3 with a progression trend before or during pregnancy; proteinuria above $3 \mathrm{~g} /$ day at any time of pregnancy, or proteinuria above $1 \mathrm{~g} /$ day at referral or in the first trimester, previous nephrotic syndrome, increase or development of proteinuria without any sign of preeclampsia, or a combination of any of these elements.

The controls were selected according to the same criteria from the Torino and Cagliari cohort. While the nephrologists' approach was very similar, in keeping with our well-established cooperation, the Torino and Cagliari Units differed with regard to the Obstetric policy towards caesarean sections (more frequently performed in Cagliari [5]), therefore this outcome was not considered in the present study.

\section{Statistical analysis}

Descriptive analysis was performed as appropriate (mean and standard deviation for parametric and median and range for non-parametric data). Paired $T$ test, Chi-square test, Fisher's test, Mid-p test, and Wilcoxon's test were used for comparisons between patients and controls and to evaluate the differences from referral to delivery in patients and controls. Significance was set at $<0.05$.

Statistical evaluation was performed using SPSS vers18.0 for Windows (SPSS Chicago Ill, USA).

\section{Ethical issues}

Systematic counselling about the diet was provided. Patients were informed that few data on the supplemented diet during pregnancy were available outside of our group, furthermore, the limits and goals of the lowprotein diets were extensively discussed. The importance of timely reporting of side effects or doubts was underlined; a written schema, progressively updated, was supplied. The first version is available elsewhere [5]. The most recent update is available in the Appendix. 
The study was approved by the Ethics committee of the OIRM Sant'Anna $\left(\mathrm{n}^{\circ}\right.$ pratica $335 ; \mathrm{n}^{\circ}$ protocollo 11551/c28.2 del 4/3/2011). All patients signed a dedicate informed consent.

\section{Results}

\section{Baseline data}

The main baseline data of the 36 patients who followed the diet for at least one month and of the 31 patients who delivered a live-born singleton baby (excluded: 3 twin deliveries, 1 pregnancy termination following the mother's wishes, 1 spontaneous miscarriage) are reported in Table 1. Two patients in the on-diet group undertook two pregnancies.

Table 2 reports the baseline data in the control group of 47 pregnancies homogeneously selected according in Torino and Cagliari; there were 42 singleton deliveries and 5 spontaneous miscarriages.

The two groups are homogeneous with regard to the main clinical parameters: age (singletons only: on diet: 33 vs controls 33.5 years); and referral week (7 vs 9 weeks). CKD stage was non significantly lower in on diet patients (CKD 3-5: $48.4 \%$ vs $64.3 \%$, p: 0.26 ), conversely, prevalence of hypertension was non significantly higher $(51.6 \%$ vs $40.5 \%$, p: 0.48$)$. Nephrotic range proteinuria ( $16.1 \%$ vs $12.2 \%$, p 0.74$)$ was also non significantly higher in on diet patients. The combination of hypertension and proteinuria was present in 14/36 $(38.9 \%)$ on-diet patients and in 14/47 (29.8\%) controls $(p=0.35)$. There were more diabetic nephropathies in ondiet patients (on diet: $31 \%$ vs controls: $5.3 \%$; p: 0.007) while lupus nephropathies were non-significantly higher in controls (on diet: $10.3 \%$ vs controls $23.7 \%$; p: 0.28 (Fisher)), presumably as a reflection of the referral pattern of the individual Nephrology Units.

\section{Pregnancy outcomes: kidney function and proteinuria}

All of the patients on the diet followed it throughout pregnancy; no diet- or supplement- related side effects were reported and abdominal discomfort, when present, was not considered related to the diet itself. According to dietary recall, compliance was good; however, especially in the second period, in which the diet was more detailed and no more merely qualitative, some patients complained that it was very intrusive in their daily life.

An increase in serum creatinine leading to a shift towards a higher CKD stage was observed in $19.4 \%$ ondiet and $9.5 \%$ controls (p: 0.2 (Fisher)).

Proteinuria increased significantly in both patients and controls (new onset or doubling of proteinuria: $54.8 \%$ of on-diet subjects and $50 \%$ of controls; p: 0.5 (Fisher)). However, serum albumin and total proteins only moderately, and non significantly decreased at the end of pregnancy (diet group: total proteins: $6.5 \mathrm{~g} / \mathrm{dL}$ at start vs
$5.7 \mathrm{~g} / \mathrm{dL}$ at delivery, albumin $3.75 \mathrm{~g} / \mathrm{dL}$ at start vs $2.9 \mathrm{~g} /$ $\mathrm{dL}$ at delivery; control group: total proteins: $6.5 \mathrm{vs} 6.1 \mathrm{~g} /$ $\mathrm{dL}$, albumin $3.4 \mathrm{vs} 3.24 \mathrm{~g} / \mathrm{dL}$ ) (Tables 3 and 4 ).

At 3 months after delivery serum creatinine increased and GFR decreased in both groups, in keeping with the reversal of pregnancy-related hyperfiltration. The decrease in proteinuria is probably due both to the reversal of the hyperflitraton phase, but other less known pregnancyrelated permeability changes mechanisms may also play a role (Tables 3 and 4), Figs. 1, 2 and 3.

\section{Pregnancy outcomes: prevalence of small for gestational} age and preterm babies

Tables 5 and 6 report the main data regarding birthweight and timing of delivery in on-diet patients and in controls. No significant differences were observed for the overall prevalence of preterm delivery $(<37$ completed gestational weeks), which was over $70 \%$ in both groups (ondiet singletons $77.4 \%$; controls $71.4 \%$; p: 0.76 ), or in the prevalence of children with birth-weight at or below 2.5 Kg (21/31: 66.7 \% vs 25/42: $59.5 \%$, p: 0.32 ).

The Figs. 4 and 5, based upon the original Parazzini charts that were the most commonly used references in Italy throughout the study period, summarize the relationship between birth-weight and prematurity in the two settings. Early preterm delivery (on diet: $32.3 \%$ vs controls: $35.7 \%$ ) and extremely low birth-weight (on diet: $9.7 \%$ vs controls: $23.8 \%$ ) were more common in control groups, and the only two extremely preterm deliveries were observed in the control group (p: 0.505).

The birth-weighty centiles, assessed by the Parazzini chart, reference in most of the period of study, showed a lower prevalence of babies below the 10th centile or extremely preterm (below 28 weeks) in on diet patients versus in controls; the difference (one tailed Fisher exact test) reaches statistical significance $(12.9 \%$ vs $33.3 \%$ p: 0.04). If centiles are calculated with INeS charts, the figures are similar (below 10th centile: $9.7 \%$ on diet vs $26.2 \%$ controls, but the difference doesn't reach statistical significance (p: 0.068)).

Conversely, gestational age and birth weight did not differ in the two cohorts (Tables 5 and 6 and Figs. 1 and 2 ). One twin child diet of on-diet mother died (cerebral haemorrhage after heart surgery for cardiac malformation); none of the singletons died in the on-diet series, while two perinatal deaths occurred in the control group $(p=0.505)$.

\section{Discussion}

An often-cited quote by Feuerbach states: "a man is what he eats"; indeed there are good reasons to reflect on Feuerbach's clever and polemic sentence in the era of epigenetics and of rediscovery of the importance of what we eat to prevent diseases and possibly to cure them. 
Table 1 Baseline data: "On-diet": 36 pregnancies in patients who followed a supplemented vegan diet in pregnancy

(31 singleton deliveries)

\begin{tabular}{|c|c|c|c|c|c|c|c|c|c|c|}
\hline Case & $\begin{array}{l}\text { Age } \\
\text { (yrs) }\end{array}$ & $\begin{array}{l}\text { Pre-conceptional; } \\
\text { Referral-week }\end{array}$ & Kidney disease & $\begin{array}{l}\mathrm{s} C r \mathrm{mg} / \mathrm{dL} \text { (EPI-GFR } \\
\mathrm{mL} / \mathrm{min})\end{array}$ & $\begin{array}{l}\text { CKD } \\
\text { stage }\end{array}$ & $\begin{array}{l}\text { PtU } \\
(g / 24 h)\end{array}$ & $\begin{array}{l}\text { Pt/Alb } \\
\text { (g/dL) }\end{array}$ & HT & $\begin{array}{l}\text { Therapy } \\
\text { at referral }\end{array}$ & BMI \\
\hline 1 & 35 & pre; ICSI & Diab neph & $1.2(59)$ & 3 & 2.5 & $5.9 / 2.8$ & Yes & Insulin, doxazosine & 23.5 \\
\hline 2 & 35 & pre; $8 w$ & Diab neph & $1.6(45)$ & 4 & 1.8 & $6.5 / 3.8$ & No & Insulin & 22 \\
\hline 3 & 28 & $7 w$ & Sponge kidney & $3.2(19)$ & 4 & 0.8 & $6.5 / 3.5$ & No & EPO, Vit. D & 22 \\
\hline 4 & 37 & pre; $6 \mathrm{w}$ & Diab neph & $1.2(58)$ & 3 & 5.9 & $5.8 / 3.2$ & No & Insulin & 22 \\
\hline 5 & 32 & $6 w$ & SLE & $0.7(115)$ & 1 & 2.7 & $6.0 / 3.1$ & Yes & Pred., ASA, omeprazole, a-MD & 24 \\
\hline 6 & 35 & pre; $7 w$ & Reflux & $3.2(18)$ & 4 & 1.0 & $8.4 / 4.5$ & Yes & Vit. D, b-blocker ASA & 19 \\
\hline 7 & 29 & $9 w$ & Diab neph & $1.5(47)$ & 3 & 6.3 & $6.6 / 3.6$ & Yes & Insulin, nifedipine & 20 \\
\hline 8 & 38 & $17 w$ & fibrillary GN & $0.6(116)$ & 1 & 3.6 & $5.8 / 2.6$ & No & None & 22 \\
\hline 9 & 32 & $6 w$ & Kidney graft & $1.2(60)$ & 2 & 0.5 & $6.9 / 4.0$ & No & Pred., CyA, ranitidine, ASA, & 24 \\
\hline 10 & 20 & $5 w$ & SLE & $0.6(132)$ & 1 & 2.5 & $6.8 / 3.3$ & No & Pred. & 21 \\
\hline 11 & 37 & $7 w$ & Kidney graft & $1.5(44)$ & 3 & 0.8 & $7.0 / 3.9$ & Yes & $\begin{array}{l}\text { Pred., CyA, VitD, nifedipine, ASA EPO, } \\
\text { ranitidine }\end{array}$ & 27 \\
\hline 12 & 30 & $6 w$ & $\lg A \mathrm{GN}$ & $1.3(55)$ & 3 & 0.7 & $6.9 / 4.1$ & No & Levothyroxine & 18.9 \\
\hline 13 & 28 & $10 w$ & $\lg A$ GN & $1(77)$ & 2 & 2 & $6.6 / 3.7$ & No & None & 19.9 \\
\hline 14 & 36 & pre; $5 \mathrm{w}$ & Diab neph & $1(68)$ & 2 & 0.6 & $6.4 / 4.1$ & Yes & $\begin{array}{l}\text { Lansoprazole, levothyroxine, ASA, } \\
\text { Niphedipine, insulin, }\end{array}$ & 21.5 \\
\hline 15 & 35 & $7 w$ & Diab neph & $1.2(56)$ & 3 & 0.7 & $7.4 / 4.3$ & No & Insulin & 18.2 \\
\hline 16 & 40 & $24 w$ & Diab neph & $0.9(76)$ & 2 & 3.1 & $6.7 / 3.3$ & Yes & Insulin, levothyroxine, Nifedipine & 24 \\
\hline 17 & 36 & $20 w$ & $\lg A$ GN & $1.1(64)$ & 2 & 2.4 & $5.6 / 4.1$ & No & None & 22.3 \\
\hline 18 & 36 & pre; $7 w$ & SLE & $2.9(20)$ & 4 & 3.4 & $6.5 / 3.9$ & Yes & Pred., levothyroxine, a-MD & 24.5 \\
\hline 19 & 38 & $6 w$ & FSGS & $0.6(116)$ & 1 & 2.1 & $6.3 / 3.3$ & No & CyA & 25 \\
\hline 20 & 33 & pre; $8 w$ & Kidney graft & $1.3(52)$ & 3 & 0.2 & $7.2 / 3.9$ & Yes & Pred, TAC & 30 \\
\hline 21 & 31 & pre; $9 w$ & Sponge kidney & $1.6(43.3)$ & 3 & 0.3 & $6.5 / 4.0$ & Yes & ASA, a-MD & 23.4 \\
\hline 22 & 33 & pre; $7 w$ & Reflux & $0.7(110.4)$ & 1 & 0.8 & $6.0 / 3.7$ & Yes & ASA, b-bloc & 21.8 \\
\hline 23 & 38 & pre; $6 \mathrm{w}$ & Pyelonephritis & $1.2(59)$ & 3 & 0.2 & $6.5 / 3.9$ & Yes & $\mathrm{a}-\mathrm{MD}$ & 19.7 \\
\hline 24 & 26 & $5 w$ & $\begin{array}{l}\text { Single kidney, } \\
\text { previous HUS }\end{array}$ & $1(78)$ & 2 & 0.3 & $6.8 / 3.8$ & Yes & ASA & 23.4 \\
\hline 25 & 41 & pre; $7 w$ & GN & $0.8(86.6)$ & 2 & 0.8 & $7.2 / 3.8$ & Yes & ASA & 33.6 \\
\hline 26 & 32 & $6 w$ & $\lg A$ GN & $1(74.7)$ & 2 & 0.6 & $6.8 / 3.9$ & No & ASA & 24.9 \\
\hline 27 & 36 & $18 w$ & Diab neph & $0.7(106.3)$ & 1 & 0.9 & $7.4 / 4.4$ & Yes & ASA, a-MD, Insulin & 34 \\
\hline 28 & 33 & $27 w$ & LLAC & $0.4(136.2)$ & 1 & 1.3 & $6.0 / 3.6$ & Yes & $\mathrm{a}-\mathrm{MD}$ & 29.7 \\
\hline 29 & 33 & $14 w$ & Unknown & $0.8(105.6)$ & 1 & 2.2 & $5.7 / 3.0$ & No & ASA & 24.8 \\
\hline
\end{tabular}


Table 1 Baseline data: "On-diet": 36 pregnancies in patients who followed a supplemented vegan diet in pregnancy (31 singleton deliveries) (Continued)

\begin{tabular}{|c|c|c|c|c|c|c|c|c|c|c|}
\hline 30 & 32 & $30 w$ & Unknown & 1.7 (39.6) & 3 & 0.1 & $6.2 / 3.6$ & No & / & 26.7 \\
\hline 31 & 31 & $8 w$ & Diab neph & $1.48(47)$ & 3 & 0.1 & $6.78 / 4.68$ & No & ASA, Insulin & 19.5 \\
\hline 32 (twin) & 31 & $21 w$ & Diab neph & $0.5(128)$ & 1 & 5.4 & $5.5 / 2.8$ & No & Insulin, levothyroxine & 17.9 \\
\hline 33 (twin) & 37 & $12 w$ & Unknown & $0.7(112)$ & 1 & 0.8 & $7.1 / 3.5$ & No & None & 31.4 \\
\hline 34 (twin) & 39 & $11 w$ & Previous PNA & $0.57(184.1)$ & 1 & 0.29 & $6.11 / 3.37$ & No & ASA & 32.32 \\
\hline 35 (termination) & 26 & $18 w$ & MGN & $0.6(126)$ & 1 & 5.5 & $5.1 / 2.3$ & No & None & 19 \\
\hline 36 (miscarriage) & 37 & $7 w$ & Kidney graft & $1.7(38)$ & 3 & 0.1 & $6.9 / 3.9$ & Yes & $\begin{array}{l}\text { Pred., TAC, EPO, ASA, } \\
\text { omeprazole, Doxazosin, } \\
\text { b-bloc }\end{array}$ & 25.1 \\
\hline $\begin{array}{l}\text { Summary data all } \\
\text { cases } \\
\text { Median (min-max) }\end{array}$ & $\begin{array}{l}34 \\
(20-41)\end{array}$ & $7(5-30)$ & - & $\begin{array}{l}\mathrm{sCr} \\
1.05(0.4-3.2) \\
\mathrm{GFR}-\mathrm{EPI} \\
66.0(18.0-184.1)\end{array}$ & $2(1-4)$ & $\begin{array}{l}0.8 \\
(0.1-6.3)\end{array}$ & $\begin{array}{l}\text { Pt } \\
6.5 \\
(5.1-8.4) \\
\text { Alb } \\
3.75 \\
(2.3-4.68)\end{array}$ & $\begin{array}{l}17 / 36 \\
47.2 \%\end{array}$ & - & $\begin{array}{l}23.4 \\
(17.9-34.0)\end{array}$ \\
\hline $\begin{array}{l}\text { Summary data } \\
\text { Singletons } \\
\text { Median (min-max) }\end{array}$ & $\begin{array}{l}33 \\
(20-41)\end{array}$ & $7(5-30)$ & - & $\begin{array}{l}\mathrm{sCr} \\
1.20(0.4-3.2) \\
\mathrm{GFR}-\mathrm{EPI} \\
60.0(18.0-136.2)\end{array}$ & $3(1-4)$ & $\begin{array}{l}0.9 \\
(0.1-6.3)\end{array}$ & $\begin{array}{l}\text { Pt } \\
6.5 \\
(5.6-8.4) \\
\text { Alb } \\
3.8 \\
(2.6-4.68)\end{array}$ & $\begin{array}{l}16 / 31 \\
51.6 \%\end{array}$ & - & $\begin{array}{l}23.4 \\
(18.2-34.0)\end{array}$ \\
\hline
\end{tabular}

Data at referral: data observed at the first follow-up in our unit

$H T$ hypertension, SLE systemic lupus erythematosus, IgA GN IgA nephropathy, FSGS focal segmental glomerlosclerosis, Diab Neph diabetic nephropathy, BMI body mass index, PtU 24 hour proteinuria, sCr serum creatinine, GFR glomerular filtration rate, SLE systemic lupus erythematosus. CyA cyclosporine A, ASA acetyl salicylic acid, Pred. prednisone, TAC tacrolimus, EPO erythropoietin, B-Bloc beta blocker, a-MD alpha methyldopa, ICSI intracytoplasmatic sperm injection 
Table 2 Baseline data: "controls": 47 pregnant patients on unrestricted diet in pregnancy (22 singleton deliveries in Cagliari, 20 in Torino)

\begin{tabular}{|c|c|c|c|c|c|c|c|c|c|c|}
\hline Case & Age (yrs) & $\begin{array}{l}\text { Pre-conceptional; } \\
\text { Referral-week }\end{array}$ & Kidney disease & $\begin{array}{l}\mathrm{s} C r \mathrm{mg} / \mathrm{dL} \text { (EPI-GFR } \\
\mathrm{mL} / \mathrm{min} \text { ) }\end{array}$ & $\begin{array}{l}\text { CKD } \\
\text { stage }\end{array}$ & $\begin{array}{l}\mathrm{PtU} \\
(\mathrm{g} / 24 \mathrm{~h})\end{array}$ & $\begin{array}{l}\mathrm{Pt} / \mathrm{Alb} \\
(\mathrm{g} / \mathrm{dL})\end{array}$ & HT & Therapy at referral & $\mathrm{BMI}$ \\
\hline 1 & 33 & $11 \mathrm{w}$ & $\lg A \mathrm{GN}$ & $0.9(84.3)$ & 2 & 1.1 & $6.0 / 2.9$ & No & Pred & 20.2 \\
\hline 2 & 34 & pre; $7 w$ & SLE & $0.7(113.4)$ & 1 & 1.3 & $5.8 / 3.5$ & No & Steroids, AZA & 19.6 \\
\hline 3 & 34 & pre; $6 w$ & SLE & $0.7(113.4)$ & 1 & 1.3 & $6.3 / 2.9$ & Yes & a-MD, Pred., CyA & 20.8 \\
\hline 4 & 38 & pre; $22 w$ & Unknown & $0.8(93.8)$ & 1 & 1.7 & $\mathrm{Na}$ & Yes & ASA, Nifedipine, a-MD & 26 \\
\hline 5 & 29 & pre; $5 \mathrm{w}$ & $\lg A \mathrm{GN}$ & $0.9(86.7)$ & 2 & 2.1 & $6.3 / 3.5$ & Yes & $\mathrm{a}-\mathrm{MD}$ & 22.1 \\
\hline 6 & 26 & pre; $8 w$ & Diab neph & $0.4(144.2)$ & 1 & 2.6 & $5.6 / 3.3$ & No & ASA, Insulin & 23.4 \\
\hline 7 & 35 & pre; $7 w$ & SLE, LLAC & $0.5(129.3)$ & 1 & 3.9 & $5.2 / 2.9$ & No & Pred & 20.2 \\
\hline 8 & 19 & pre; $13 w$ & SLE & $0.6(135.6)$ & 1 & 4 & $5.9 / 3$ & No & none & 18.8 \\
\hline 9 & 36 & pre; $13 \mathrm{w}$ & FSGS & $0.9(82.5)$ & 2 & 4.2 & $5.7 / 3.1$ & Yes & ASA, a-MD & 20.3 \\
\hline 10 & 41 & pre; $6 w$ & SLE & $1(71.9)$ & 2 & 5.4 & $5.5 / 3.6$ & Yes & $\mathrm{a}-\mathrm{MD}$ & 18.3 \\
\hline 11 & 35 & pre; 20w & Diab neph & $1.2(58.7)$ & 3 & 0.1 & $\mathrm{Na}$ & Yes & a-MD, Insulin & 25.6 \\
\hline 12 & 39 & pre; $7 w$ & SLE & $1.4(47.4)$ & 3 & 0.3 & $6.5 / 3.7$ & Yes & Pred., AZA & 23 \\
\hline 13 & 32 & pre; $9 w$ & SLE & $1.4(49.8)$ & 3 & 0.8 & $6.7 / 4.5$ & Yes & Pred & 21.6 \\
\hline 14 & 35 & pre; $8 w$ & $\lg A \mathrm{GN}$ & $1.4(50)$ & 3 & 1.7 & $6.8 / 3.3$ & No & none & 24.8 \\
\hline 15 & 38 & pre; $7 w$ & Unknown & $1.6(40.6)$ & 3 & 1.4 & $6.2 / 4.3$ & No & none & 23.9 \\
\hline 16 & 31 & $6 w$ & $\lg A \mathrm{GN}$ & $1.6(42.6)$ & 3 & ++ & $7 / 3.3$ & No & none & 32.5 \\
\hline 17 & 36 & pre; $6 \mathrm{w}$ & GN & $1.8(35.7)$ & 3 & 0.4 & $7.3 / 4$ & No & none & 22.5 \\
\hline 18 & 23 & pre; $13 w$ & Unknown & 1.9 (36.6) & 3 & 2.8 & $6.1 / 3$ & No & none & 30.1 \\
\hline 19 & 30 & na & $\lg A \mathrm{GN}$ & $1.4(50)$ & 3 & 6.2 & na & Yes & $\mathrm{a}-\mathrm{MD}$ & 21.6 \\
\hline 20 & 34 & pre; $12 w$ & SLE, LLAC & $2.2(28.4)$ & 4 & 1.2 & $5.7 / 3.2$ & Yes & a-MD, ASA, EPO & 21.4 \\
\hline 21 & 28 & $7 \mathrm{w}$ & Unknown & $1.6(43.9)$ & 3 & 1.6 & $7.4 / 4.3$ & No & none & 23.7 \\
\hline 22 & 33 & pre; $12 \mathrm{w}$ & GN & $0.5(127.6)$ & 1 & 1.1 & $7 / 4$ & No & none & 21.8 \\
\hline $\begin{array}{l}\text { Summary data } \\
\text { Cagliari }\end{array}$ & $\begin{array}{l}34 \\
(19-41)\end{array}$ & $8(5-22)$ & & $\begin{array}{l}\mathrm{sCr} \\
1.1(0.4-2.2) \\
\mathrm{GFR}-\mathrm{EPI} \\
65.3(28.4-144.2)\end{array}$ & & $\begin{array}{l}1.6 \\
(0.1-6.2)\end{array}$ & $\begin{array}{l}\text { Pt } \\
6.2 \\
(5.2-7.4) \\
\text { Alb } \\
3.3 \\
(2.9-4.5)\end{array}$ & $\begin{array}{l}10 \\
45.5 \%\end{array}$ & & $\begin{array}{l}21.95 \\
(18.3-32.5)\end{array}$ \\
\hline 1 & 39 & Pre; $8 \mathrm{w}$ & Interstitial & $1.6(40)$ & 3 & 1.3 & $7.3 / 3.7$ & Yes & $\begin{array}{l}\text { Felodipine, Doxazosin, } \\
\text { Levotiroxina, ASA }\end{array}$ & 26.7 \\
\hline 2 & 27 & $14 w$ & Reflux & $1.5(47)$ & 3 & 0.3 & $6.5 / 3.4$ & No & None & 18.0 \\
\hline 3 & 34 & $20 w$ & Chronic PN & $1.5(45)$ & 3 & 2.0 & $\mathrm{Na}$ & No & None & 23.3 \\
\hline 4 & 23 & $13 w$ & $\lg A \mathrm{GN}$ & $1.3(56)$ & 3 & 0.5 & $6.4 / 3.1$ & Yes & $\mathrm{a}-\mathrm{MD}, \mathrm{ASA}$ & 22.7 \\
\hline 5 & 32 & Pre; $5 \mathrm{w}$ & $\lg A \mathrm{GN}$ & $1.2(58)$ & 3 & 0.3 & $6.1 / 3.4$ & No & Steroids, Allopurinole & 19.1 \\
\hline
\end{tabular}


Table 2 Baseline data: "controls": 47 pregnant patients on unrestricted diet in pregnancy (22 singleton deliveries in Cagliari, 20 in Torino) (Continued)

\begin{tabular}{|c|c|c|c|c|c|c|c|c|c|c|}
\hline 6 & 35 & Pre; $8 \mathrm{w}$ & $\lg A$ GN & $1.3(54)$ & 3 & 0.5 & $6.6 / 2.7$ & Yes & a-MP, Niphedipine & 24.4 \\
\hline 7 & 22 & $27 w$ & Reflux & $2.9(22)$ & 4 & 0.5 & $7.3 / 3.4$ & No & Niphedipine & 22.2 \\
\hline 8 & 39 & Pre; 14 w & Chronic PN & $1.4(47)$ & 3 & 0.2 & $8.1 / 4.0$ & Yes & B-bloc, ASA & 18.4 \\
\hline 9 & 31 & $20 w$ & Reflux & $1.3(54)$ & 3 & 0.6 & $7.2 / 3.7$ & Yes & $\begin{array}{l}\text { B-bloc, Doxazosine, Niphedipine, } \\
\text { Isosorbide }\end{array}$ & 19.5 \\
\hline 10 & 25 & $33 w$ & Reflux & $1.3(57)$ & 3 & 0.8 & $6.0 / 3.1$ & Yes & a-MP & 19.3 \\
\hline 11 & 35 & $7 ; w$ & Interstitial & $1.3(52)$ & 3 & 0.6 & $6.0 / 3.2$ & Yes & None & 25.6 \\
\hline 12 & 33 & Pre; $12 \mathrm{w}$ & Chronic PN & $1.2(60)$ & 3 & 0.1 & $6.4 / 3.2$ & No & Clonidine, a-MP, ASA & 19.7 \\
\hline 13 & 30 & $6 w$ & Kidney graft & $1.2(59)$ & 3 & 0.2 & $7.6 / 3.2$ & No & $\begin{array}{l}\text { TAC, Pred, Pantoprazole, } \\
\text { Allopurinolo }\end{array}$ & 20.3 \\
\hline 14 & 32 & $29 w$ & HIV neph. & $1.43(56)$ & 3 & 0.4 & $7.0 / 3.1$ & No & Antiretroviral therapy Omeprazole & 20.0 \\
\hline 15 & 36 & $6 w$ & Kidney graft & $1.1(56)$ & 3 & 0.1 & $6.9 / 4.7$ & No & Pred, CyA, Omeprazole, ASA & 24.7 \\
\hline 16 & 38 & Pre; 8 w & single kidney & $0.8(56)$ & 3 & 0.1 & $6.8 / 4.4$ & No & Calcium carbonate & 15.6 \\
\hline 17 & 27 & $5 w$ & SLE & $0.6(193.6)$ & 1 & 1.45 & $7.09 / 4.37$ & No & ASA, Steroids & 30.4 \\
\hline 18 & 37 & $20 w$ & FSGS & $0.7(81.6)$ & 2 & 2.33 & $6.56 / 3.62$ & No & none & 23.6 \\
\hline 19 & 26 & $12 w$ & IR e proteinuria & $1.1(101.2)$ & 1 & 2 & $6.45 / 3.49$ & No & ASA & 32.4 \\
\hline 20 & 36 & $16 w$ & PNC & $0.6(122)$ & 1 & 1.03 & $7.55 / 4.10$ & No & Thyroxine & 20.2 \\
\hline 21 (miscarriage) & 38 & $7 w$ & $\begin{array}{l}\text { Chronic PN, single } \\
\text { kidney }\end{array}$ & $1.9(31)$ & 3 & 0.1 & $7.3 / 5.0$ & No & None & 24.9) \\
\hline 22 (miscarriage) & 37 & Pre; $8 \mathrm{w}$ & Single kidney & $0.8(58)$ & 3 & 0.1 & 7.6/4.7 & No & None & 15.6 \\
\hline 23 (miscarriage) & 36 & $5 w$ & Kidney graft & $1.3(53)$ & 3 & 0.4 & $\mathrm{Na}$ & No & CyA, AZA & 25.5 \\
\hline 24 (miscarriage) & 37 & Pre; 5 w & single kidney & $0.9(55)$ & 3 & 0.1 & na & No & Calcium carbonate, & 16.2 \\
\hline 25 (miscarriage) & 30 & $9 w$ & Diab. Neph & $1.4(50)$ & 3 & 0.2 & $6.9 / 4.1$ & No & Insuline & 22.7 \\
\hline Summary data Torino & $\begin{array}{l}34 \\
(22-39)\end{array}$ & $9(5-33)$ & - & $\begin{array}{l}\mathrm{sCr} \\
1.3(0.6-2.9) \\
\text { GFR-EPI } \\
56.0(22.0-193.6)\end{array}$ & $3(1-4)$ & $\begin{array}{l}0.4 \\
(0.1- \\
2.33)\end{array}$ & $\begin{array}{l}\text { Pt } \\
6.9 \\
(6.0-8.1) \\
\text { Alb } \\
3.55 \\
(2.7-5.0)\end{array}$ & $\begin{array}{l}7 \\
28.0 \%\end{array}$ & - & $\begin{array}{l}22.2 \\
(15.6-32.4)\end{array}$ \\
\hline
\end{tabular}


Table 2 Baseline data: "controls": 47 pregnant patients on unrestricted diet in pregnancy (22 singleton deliveries in Cagliari, 20 in Torino) (Continued)

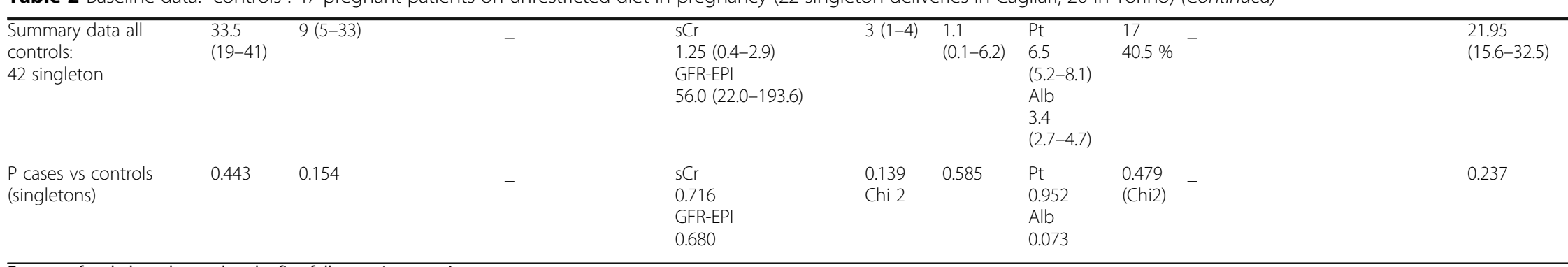

Data at referral: data observed at the first follow-up in our unit

HT hypertension, SLE systemic lupus erythematosus, IgA GN IgA nephropathy, FSGS focal segmental glomerlosclerosis, Diab Neph diabetic nephropathy, BMI body mass index, PtU 24 hour proteinuria, SCr serum

creatinine, GFR glomerular filtration rate, SLE systemic lupus (erithematosus)elim erythematosus. CyA cyclosporine A, ASA acetyl salicylic acid, Pred. prednisone, TAC tacrolimus, EPO erythropoietin, B-Bloc beta blocker, a-

con 
Table 3 Maternal data at delivery: "on-diet": 31 singleton deliveries and 3 twin deliveries

\begin{tabular}{|c|c|c|c|c|c|c|c|c|}
\hline Case & $\mathrm{sCr}$ mg/dL (EPI-GFR mL/min) & Stage CKD & $\mathrm{PtU} g / 24 \mathrm{~h}$ & Pt/Alb (g/dL) & Weight gain & Hospitalization & $\begin{array}{l}\mathrm{sCr} \mathrm{mg} / \mathrm{dL} \\
\text { (EPI-GFR mL/min) } 3 \text { months }\end{array}$ & $\begin{array}{l}\text { PtU g/die } \\
\text { Serum Alb g/dl } \\
3 \text { months }\end{array}$ \\
\hline 1 & $1.8(36)$ & 3 & 6.2 & $4.8 / 1.9$ & $9(13.4 \%)$ & 95 & $2.0(45)$ & $3 / 2.5$ \\
\hline 2 & $1.8(36)$ & 3 & 5.6 & $5.7 / 2.8$ & 11 (20 \%) & 73 & $1.9(40)$ & $4 / 3$ \\
\hline 3 & $3.7(16)$ & 4 & 2.6 & $6.3 / 3.6$ & $9(16 \%)$ & 55 & 4.5 & $0.3 / 3.6$ \\
\hline 4 & $2(31)$ & 3 & 1.9 & $5.6 / 2.9$ & $9(18 \%)$ & 47 & $2.1(21)$ & $1.5 / 3.1$ \\
\hline 5 & $0.7(115)$ & 1 & 3.4 & $4.8 / 2.9$ & $14(21.5 \%)$ & 123 & - & - \\
\hline 6 & $2.9(20)$ & 4 & 2.0 & $6.2 / 2.9$ & $10(21.7 \%)$ & 80 & $2.8(25)$ & $1.5 / 3.5$ \\
\hline 7 & $5(11)$ & 5 & 17.3 & $4.2 / 1.8$ & $16(25 \%)$ & 93 & $4.3(19)$ & $5 / 3.1$ \\
\hline 8 & $0.6(116)$ & 1 & 2.1 & $5.0 / 2.4$ & 10 (20\%) & 30 & $0.8(120)$ & $4 / 3.2$ \\
\hline 9 & $1.3(54)$ & 3 & 3.6 & $5.3 / 2.8$ & $8(12 \%)$ & 84 & $1.2(64)$ & $1.3 /-$ \\
\hline 10 & $0.5(140)$ & 1 & 2.9 & $5.4 / 2.7$ & $11(17 \%)$ & 63 & $0.7(125.1)$ & $6.2 / 2.8$ \\
\hline 11 & $1.8(35)$ & 3 & 5.4 & $5.4 / 2.8$ & $5(7 \%)$ & 99 & $1.7(52.9)$ & $6.8 / 3.8$ \\
\hline 12 & $0.8(99)$ & 1 & 5.7 & $5.5 / 2.7$ & 10 (17.9\%) & 9 & $1.2(60.6)$ & $5.7 / 3.2$ \\
\hline 13 & $1.5(45)$ & 3 & 5.5 & $5.0 / 2.6$ & 4 (7.8 \%) & 28 & $2.9(23.3)$ & $3.4 / 4$ \\
\hline 14 & $1(73)$ & 2 & 4.7 & $4.5 / 2.2$ & 12 (21\%) & 29 & $0.9(82.5)$ & $4.4 / 2.2$ \\
\hline 15 & $1.5(44)$ & 3 & 9.4 & $5.5 / 2.6$ & $8(14 \%)$ & 24 & 1.9 (33.7) & $1.3 / 5.7$ \\
\hline 16 & $1(72)$ & 2 & 4.4 & $6.4 / 2.8$ & 14 (30.4 \%\%) & 24 & $0.9(80.2)$ & $1.4 / 3.5$ \\
\hline 17 & $1.1(65)$ & 2 & 2.2 & $6.0 / 2.9$ & 11 (20.4\%) & 9 & na & na \\
\hline 18 & $3.6(15)$ & 4 & 3.4 & $5.8 / 3.2$ & 12 (17.9\%) & 26 & $3.1(18.5)$ & $1.3 / 3.2$ \\
\hline 19 & $0.6(115)$ & 1 & 1.4 & $5.2 / 2.6$ & 10 (14.1\%) & 16 & $0.7(110.3)$ & $2.5 / 2.7$ \\
\hline 20 & $1.8(37)$ & 3 & 0.8 & $6.7 / 3.3$ & 7 (9.2 \%) & 12 & 1.7 (39.1) & $1 / 3.9$ \\
\hline 21 & $1.2(60)$ & 2 & 1.7 & $5.9 / 3.1$ & 15 (23.8 \%) & 8 & $1.8(37.2)$ & $0.3 / 4.1$ \\
\hline 22 & 0.7 (105) & 1 & 0.9 & $5.7 / 2.9$ & $10(20.4 \%)$ & 3 & $0.8(98.7)$ & $0.8 / 4.5$ \\
\hline 23 & $1(69)$ & 2 & 0.3 & $6.8 / 3.6$ & 12 (22.6 \%) & 4 & $1.3(53.7)$ & $0.1 / 3.7$ \\
\hline 24 & $1.2(63.2)$ & 2 & 0.6 & $6.7 / 3.6$ & 6 (10 \%) & 13 & $1.1(69.5)$ & $0.1 / 4$ \\
\hline 25 & $0.8(90.5)$ & 1 & 1.8 & $5.5 / 3.2$ & $6(7 \%)$ & 7 & 0.9 (76.6) & $0.7 / 4.3$ \\
\hline 26 & $1(75.6)$ & 2 & 0.8 & $6.6 / 3.1$ & 22 (31\%) & 5 & $1.0(74.7)$ & $0.8 / 3.3$ \\
\hline 27 & $0.9(86)$ & 2 & 0.9 & $5.9 / 3.1$ & 1 (1 \%) & 16 & na & na \\
\hline 28 & $0.5(131.1)$ & 1 & 0.4 & $6.0 / 2.7$ & $-2(-2.5 \%)$ & 5 & $0.6(118.9)$ & $0.5 / 4.7$ \\
\hline 29 & $1(89.9)$ & 2 & 6.2 & $5.5 / 2.9$ & 6 (7.9 \%) & 5 & 1.0 & na \\
\hline 30 & $2(31.6)$ & 3 & 0.1 & $6.4 / 3.2$ & 9 (15 \%) & 22 & na & na \\
\hline 31 & $2.33(27)$ & 4 & 0.41 & $6.4 / 3.3$ & 15 (22 \%) & 24 & na & na \\
\hline
\end{tabular}


Table 3 Maternal data at delivery: "on-diet": 31 singleton deliveries and 3 twin deliveries (Continued)

\begin{tabular}{|c|c|c|c|c|c|c|c|c|}
\hline 32 (twin) & $0.7(117)$ & 1 & 11.8 & $4.1 / 1.8$ & $21(42 \%)$ & 76 & $0.6(121.8)$ & $1.5 / 3.7$ \\
\hline 33 (twin) & $0.9(81.4)$ & 2 & na & $6.1 / 3.3$ & $3(3.3 \%)$ & 8 & na & na \\
\hline 34 (twin) & $0.6(120)$ & 1 & 0.9 & $5.6 / 3.0$ & $24(27.3 \%)$ & 14 & na & na \\
\hline Summary data (singletons) & $\begin{array}{l}\mathrm{sCr} \\
1.2(0.5-5.0) \\
\text { GFR-EPI } \\
63.2(11.0-140.0)\end{array}$ & $3(1-4)$ & $2.2(0.1-17.3)$ & $\begin{array}{l}\mathrm{Pt} \\
5.7(4.2-6.8) \\
\text { Alb } \\
2.9(1.8-3.6)\end{array}$ & $10.0(-2-22)$ & $24(3-123)$ & $\begin{array}{l}\mathrm{sCr} \\
1.25(0.6-4.5) \\
\text { GFR-EPI } \\
57.15(18.5-125.1)\end{array}$ & $\begin{array}{l}\text { PtU } \\
1.4(0.1-6.8) \\
\text { Serum Alb } \\
3.5(2.2-5.7)\end{array}$ \\
\hline
\end{tabular}

Legend: Data at delivery: data observed at the last control before delivery (usually at hospitalization

PtU 24 hour proteinuria, $s C r$ serum creatinine, GFR glomerular filtration rate, Alb serum albumin, na non available 
Table 4 Maternal data at delivery: "controls": 42 singleton deliveries

\begin{tabular}{|c|c|c|c|c|c|c|c|}
\hline Case & sCr mg/dL (EPI-GFR mL/min) & Stage CKD & $\mathrm{PtU} \mathrm{g} / 24 \mathrm{~h}$ & Pt/Alb (g/dL) & Weight gain $(\mathrm{Kg})$ & $\begin{array}{l}\mathrm{sCr} \mathrm{mg} / \mathrm{dL} \\
\text { (EPI-GFR mL/min) } 3 \text { months }\end{array}$ & $\begin{array}{l}\text { PtU g/die } \\
\text { Serum Alb g/dl } \\
3 \text { months }\end{array}$ \\
\hline 1 & $0.9(85)$ & 2 & 1.5 & $5.3 / 2.9$ & 10 & $0.9(85)$ & $1.7 / 3.7$ \\
\hline 2 & $0.8(96)$ & 1 & 7.2 & $6.9 / 3.3$ & 10 & $0.8(96)$ & $0.8 / 4$ \\
\hline 3 & $0.7(113)$ & 1 & 8.8 & $6.3 / 3.1$ & 10 & $0.8(96)$ & $0.4 / 3.8$ \\
\hline 4 & $0.8(94)$ & 1 & 1.5 & na & na & na & na \\
\hline 5 & $0.9(86)$ & 2 & 1.1 & $5.7 / 2.9$ & 15 & $1(76)$ & $1.3 / 3.7$ \\
\hline 6 & $0.5(136)$ & 1 & 0.8 & $5.9 / 2.9$ & 14 & $0.5(129)$ & $0.4 / 4.3$ \\
\hline 7 & $0.5(124)$ & 1 & 4.0 & $5.4 / 2.5$ & 14 & $0.5(125)$ & $1.4 / 3.2$ \\
\hline 8 & $0.5(141)$ & 1 & 3.7 & $5.7 / 3.0$ & 10 & $0.6(130)$ & $3.1 / 4.3$ \\
\hline 9 & $1.0(72)$ & 2 & 6.2 & $5.1 / 2.8$ & 18 & $1.1(64)$ & $3.5 / 3.7$ \\
\hline 10 & $1.3(51)$ & 3 & 7.9 & $4.7 / 2$ & 12 & $1.3(51)$ & $1.3 / 3.1$ \\
\hline 11 & $2.3(27)$ & 4 & 8.3 & na & na & na & na \\
\hline 12 & $1.4(47)$ & 3 & 2.5 & $6 / 3.3$ & 14 & $1.5(43)$ & $2.1 / \mathrm{na}$ \\
\hline 13 & $1.4(50)$ & 3 & 6.3 & $5.2 / 3.1$ & 13 & $1.4(49)$ & $1 / 4.1$ \\
\hline 14 & $1.4(48)$ & 3 & 3.6 & $6.4 / 3.0$ & 18 & $1.5(44)$ & $3.2 / \mathrm{na}$ \\
\hline 15 & $1.8(35)$ & 3 & 4.4 & $5.6 / 2.8$ & 11 & $1.9(33)$ & $5.7 / 3.3$ \\
\hline 16 & $1.6(42)$ & 3 & 1.8 & $6.1 / 3.0$ & 8 & $1.3(55)$ & $2.4 / 3.9$ \\
\hline 17 & 1.7 (38) & 3 & 5.6 & $5.9 / 2.9$ & 9 & $1.8(35)$ & $5.7 / 3.6$ \\
\hline 18 & $1.7(42)$ & 3 & 5.6 & $6.2 / 3.3$ & 7 & $1.9(36)$ & $8 / 4$ \\
\hline 19 & $1.4(50)$ & 3 & 6.2 & $5.3 / 3.3$ & 8 & $0.9(131)$ & $1.6 / 4.1$ \\
\hline 20 & $2.0(32)$ & 3 & 5.1 & $5.7 / 3.2$ & 4 & $1.8(37)$ & $5.4 / 3$ \\
\hline 21 & $2.3(27.8)$ & 4 & 7.1 & $6.8 / 3.1$ & 10 & $2.4(26)$ & $2.7 / 4.2$ \\
\hline 22 & $0.5(127)$ & 1 & 0.5 & $7.5 / 3.8$ & 17 & $0.7(114)$ & $0.8 / 4.6$ \\
\hline Summary data (Cagliari) & $\begin{array}{l}\mathrm{sCr} \\
1.35(0.5-2.3) \\
\mathrm{GFR}-\mathrm{EPI} \\
50.5(27.0-141.0)\end{array}$ & $3(1-4)$ & $4.75(0.5-8.8)$ & $\begin{array}{l}\mathrm{Pt} \\
5.8(4.7-7.5) \\
\text { Alb } \\
3.0(2.0-3.8)\end{array}$ & $10.5(4-18)$ & $\begin{array}{l}\mathrm{sCr} \\
1.20(0.5-2.4) \\
\text { GFR-EPI } \\
59.5(26.0-131.0)\end{array}$ & $\begin{array}{l}\text { PtU } \\
1.90(0.4-8.0) \\
\text { Serum Alb } \\
3.85(3.0-4.6)\end{array}$ \\
\hline 1 & $0.83(121)$ & 1 & 1.08 & $6.1 / 3.8$ & 8 & $1.2(57)$ & $1.0 / 3.5$ \\
\hline 2 & $0.69(80.5)$ & 2 & 1.61 & $6.1 / 2.9$ & 12 & $1.4(66)$ & $0.9 / 3.7$ \\
\hline 3 & $1.06(86)$ & 2 & 2.83 & 6.343 .3 & 10 & $1.7(39)$ & $1.2 / \mathrm{ns}$ \\
\hline 4 & $0.59(112)$ & 1 & 0.1 & $7.01 / 3.58$ & 8 & $1.9(42)$ & $2.5 / 3.2$ \\
\hline 5 & $0.83(121)$ & 1 & 1.08 & $6.08 / 3.80$ & 8 & $1.2(52)$ & $0.8 / 3.7$ \\
\hline 6 & $0.69(80.5)$ & 2 & 1.61 & $6.11 / 2.95$ & 10 & $\mathrm{Na}$ & na \\
\hline
\end{tabular}


Table 4 Maternal data at delivery: "controls": 42 singleton deliveries (Continued)

\begin{tabular}{|c|c|c|c|c|c|c|c|}
\hline 7 & $1.06(86)$ & 2 & 2.83 & $6.34 / 3.28$ & 2 & $5.2(11)$ & na \\
\hline 8 & 0.59 (112) & 1 & 0.1 & $7.01 / 3.58$ & 6 & $2.1(29)$ & $0.5 / 4.0$ \\
\hline 9 & $0.83(121)$ & 1 & 1.08 & $6.08 / 3.80$ & 11 & $2.2(29)$ & $1.2 / 3.7$ \\
\hline 10 & $0.69(80.5)$ & 2 & 1.61 & $6.11 / 2.95$ & 19 & $1.3(57)$ & $0.7 / 4.1$ \\
\hline 11 & $1.06(86)$ & 2 & 2.83 & $6.34 / 3.28$ & 11 & $1.1(62)$ & $0.1 / 4.1$ \\
\hline 12 & $0.59(112)$ & 1 & 0.1 & $7.01 / 3.58$ & 15 & $1.5(45)$ & $0.2 / 3.7$ \\
\hline 13 & $0.83(121)$ & 1 & 1.08 & $6.08 / 3.8$ & 14 & $0.9(85)$ & $0.3 / 3.8$ \\
\hline 14 & $0.69(80.5)$ & 2 & 1.61 & $6.11 / 2.95$ & 7 & $1.2(72)$ & na \\
\hline 15 & $1.06(86)$ & 2 & 2.83 & $6.34 / 3.28$ & 3 & na & na \\
\hline 16 & $0.59(112)$ & 1 & 0.1 & $7.01 / 3.58$ & 13 & $0.9\left(55^{\mathrm{a}}\right)$ & $0.1 / 4.2$ \\
\hline 17 & $0.83(121)$ & 1 & 1.08 & $6.08 / 3.80$ & 14 & na & na \\
\hline 18 & $0.69(80.5)$ & 2 & 1.61 & $6.11 / 2.95$ & 3 & na & na \\
\hline 19 & $1.06(86)$ & 2 & 2.83 & $6.34 / 3.28$ & 2 & na & na \\
\hline 20 & 0.59 (112) & 1 & 0.1 & $7.01 / 3.58$ & 19 & na & na \\
\hline $\begin{array}{l}\text { Summary data } \\
\text { Torino }\end{array}$ & $\begin{array}{l}\mathrm{sCr} \\
0.76(0.59-1,06) \\
\mathrm{GFR}-\mathrm{EPI} \\
99.0(80.5-121.0)\end{array}$ & & $1.34(0.1-2.83)$ & $\begin{array}{l}\text { Pt } \\
6.2(6.08-7.01) \\
\text { Alb } \\
3.44(2.9-3.8)\end{array}$ & $10.0(2-19)$ & $\begin{array}{l}\mathrm{sCr} \\
1.35(0.9-5.2) \\
\mathrm{GFR}-\mathrm{EPI} \\
53.5(11.0-85.0)\end{array}$ & $\begin{array}{l}\text { PtU } \\
0.75(0.1-2.5) \\
\text { Serum Alb } \\
3.7(3.2-4.2)\end{array}$ \\
\hline Summary data, all & $\begin{array}{l}\mathrm{sCr} \\
0.83(0.5-2.3) \\
\text { GFR-EPI } \\
86.0(27.0-141.0)\end{array}$ & & $2.15(0.1-8.8)$ & $\begin{array}{l}\text { Pt } \\
6.1(4.7-7.5) \\
\text { Alb } \\
3.24(2.0-3.8)\end{array}$ & $10.0(2-19)$ & $\begin{array}{l}\mathrm{sCr} \\
1.3(0.5-5.2) \\
\text { GFR-EPI } \\
55.0(11.0-131.0)\end{array}$ & $\begin{array}{l}\text { PtU } \\
1.25 \text { (0.1-8.0) } \\
\text { Serum Alb } \\
3.8(3.0-4.6)\end{array}$ \\
\hline P controls vs on diet & $\begin{array}{l}\mathrm{sCr} \\
0.018 \\
\text { GFR-EPI } \\
0.018\end{array}$ & $\begin{array}{l}0.390 \\
\text { (Chi2) }\end{array}$ & 0.876 & 0.010 & 0.364 & $\begin{array}{l}\mathrm{sCr} \\
0.565 \\
\text { GFR-EPI } \\
0.813\end{array}$ & $\begin{array}{l}\text { PtU } \\
0.499 \\
\text { Serum Alb } \\
0.074\end{array}$ \\
\hline
\end{tabular}

Legend: Data at delivery: data observed at the last control before delivery (usually at hospitalization

PtU 24 hour proteinuria, sCr serum creatinine, GFR glomerular filtration rate, Alb serum albumin, na non available

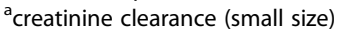



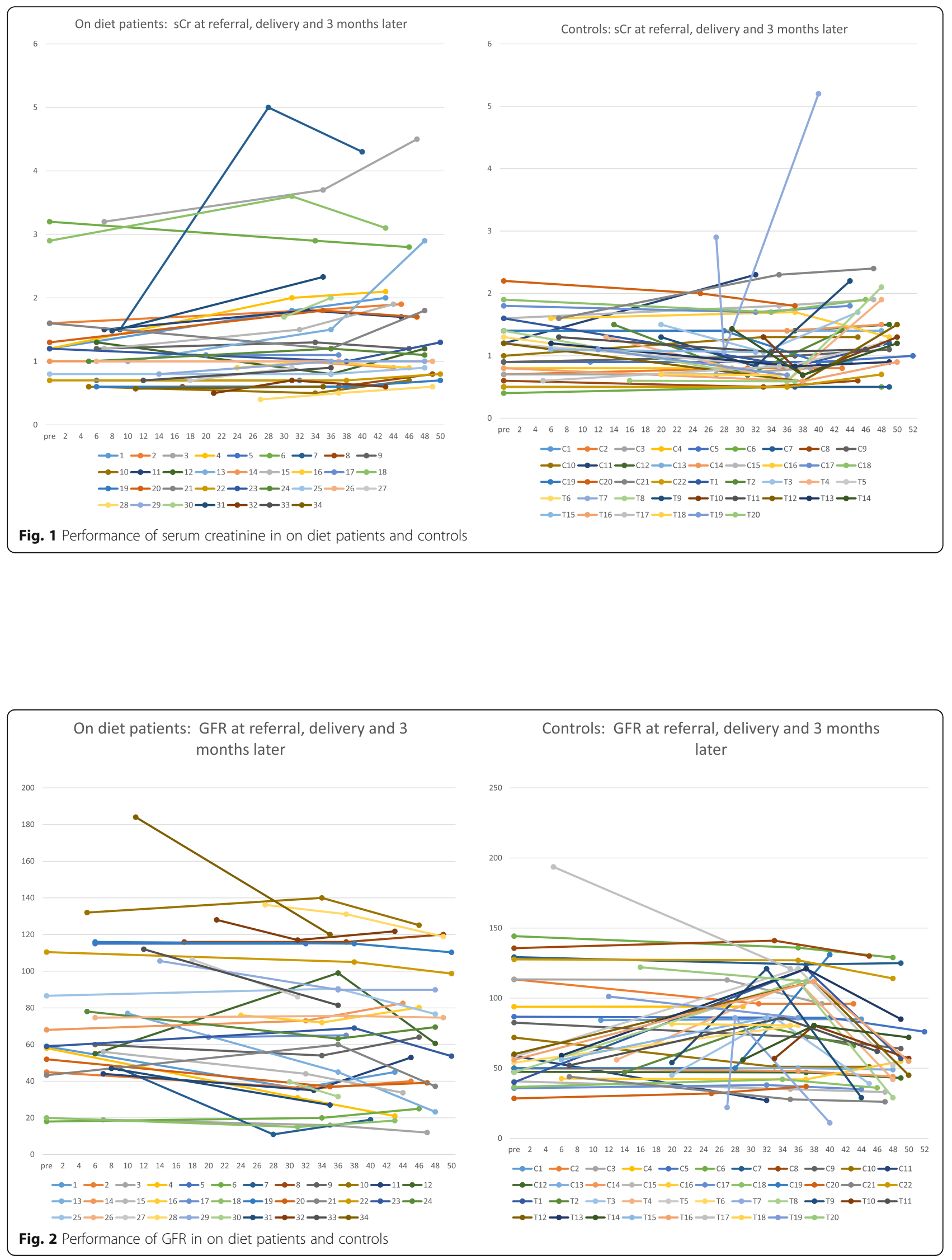


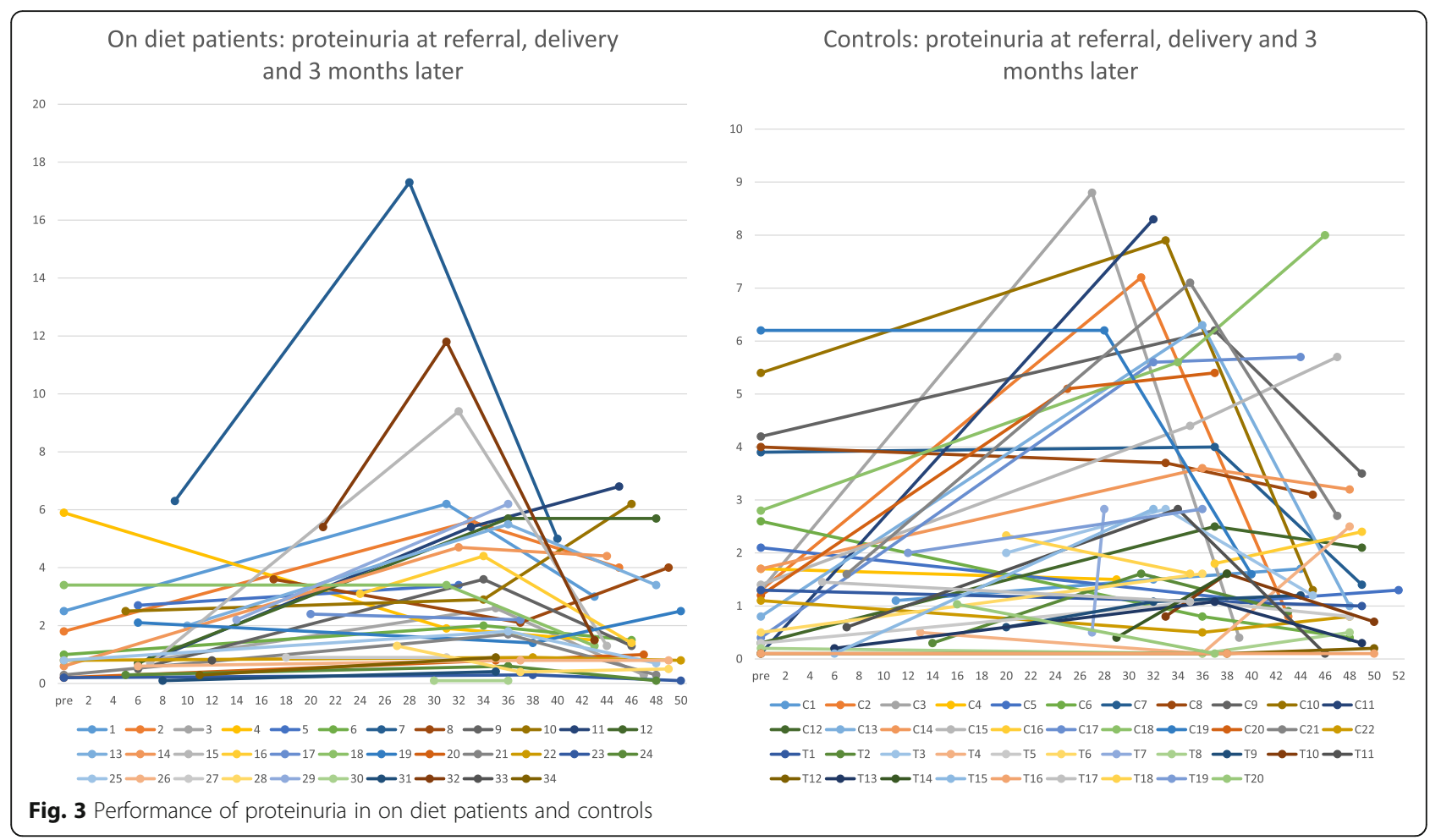

Low protein diets are a well-known tool for contrasting absolute or relative hyperfiltration in the case of nephrotic syndrome or diabetes, and in the remnant nephrons in CKD patients [14-16, 39-41]. Pregnancy is another wellacknowledged condition of physiological hyperfiltration, which may exert a negative effect on kidney function or increase proteinuria in CKD patients [42-47].

Control of hyperfiltration and of proteinuria were the potential advantages we hoped to achieve by a lowprotein diet in pregnancy, when this experience started, at a time when pregnancy in CKD was often discouraged and the common practice was to increase protein intake in pregnancy [48].

Almost unexpectedly, the finding of equivalent or better foetal growth in on-diet patients shifted our attention from the maternal kidneys to the maternal-foetal exchanges, suggesting a potential effect on the uteroplacental axis [4]. While the low numbers, and the lack of a homogeneous control group limited the interest in our findings, this larger cohort with a well-matched larger control group may allow us to refine the previous results.

Similarly to our previous studies, in the present series there is a trend towards better preserved foetal growth, that reaches statistical significance for the combined outcome of extremely preterm delivery and small for gestational age baby (below the 10th centile) (Tables 5 and 6). Preterm delivery was over $70 \%$ in cases and controls, witnessing the relevance of the renal impairment; such prevalence is in line with available studies on patients with advanced CKD [3, 5, 49-51].

In our analysis the differences between cases and controls regard the "harder" and partially overlapping outcomes, which include early preterm delivery, small for gestational age (SGA) and extreme preterm babies, "very small" babies (birth-weight is at or below1,500 g). The lower incidence of SGA has to be contextualised with the similar incidence of early preterm delivery ( $32.3 \%$ vs $35.7 \%$ in controls), since SGA is a reason for anticipating delivery [52]. This reinforces our previous findings, of a better foetal growth in children of on-diet CKD mothers (Figs. 1 and 2, Tables 5 and 6).

Our study has several limitations, which are partly shared by other studies on pregnancy: first of all, it is not randomised. However, randomization of the diet is hardly feasible outside of pregnancy and may be ethically unsound in pregnancy.

Secondly, we deal with a small number of patients, even if ours is the only study to date dedicated to this issue in CKD pregnancies.

Further research, involving a greater number of subjects is needed to highlight the differences suggested by our studies and to analyse placental vascularization and development, thus possibly offering insights into the pathogenesis of adverse pregnancy-related outcomes in CKD mothers. Theoretically, a positive effect could be due to a decrease in "vaso-toxic" elements or to an increase in "vaso-protective" ones; both are present in 
Table 5 Main Maternal-foetal outcomes and intrauterine growth: "on-diet": 31 singleton deliveries and 3 twin deliveries

\begin{tabular}{|c|c|c|c|c|c|c|c|c|}
\hline Case & $\begin{array}{l}\text { Gestational age } \\
\text { Weeks (days) }\end{array}$ & $\begin{array}{l}\text { Type of } \\
\text { delivery }\end{array}$ & $\begin{array}{l}\text { Sex of the } \\
\text { baby }\end{array}$ & Weight (g) & Centile (Parazzini) & Centile (INeS) & $\begin{array}{l}\text { Apgar } \\
(1-5 \mathrm{~min})\end{array}$ & $\mathrm{NICU}$ \\
\hline 1 & $31+0(217)$ & Vaginal & $M$ & 1595 & $50-90$ & 55 & $7-8$ & Yes \\
\hline 2 & $33+3(234)$ & CS & $\mathrm{F}$ & 1980 & $50-90$ & 63 & $9-9$ & Yes \\
\hline 3 & $35+2(247)$ & CS & $\mathrm{F}$ & 1685 & $<5$ & 5 & $8-9$ & Yes \\
\hline 4 & $31+0(217)$ & CS & M & 1970 & $50-90$ & 92 & $8-8$ & Yes \\
\hline 5 & $32+6(230)$ & CS & M & 2080 & $50-90$ & 75 & $9-9$ & No \\
\hline 6 & $34+1$ (239) & CS & $\mathrm{F}$ & 1410 & $<5$ & 3 & $8-8$ & Yes \\
\hline 7 & $28+1(197)$ & CS & $\mathrm{F}$ & 935 & $10-50$ & 42 & $7-8$ & Yes \\
\hline 8 & $37+1(260)$ & Vaginal & M & 2620 & $10-50$ & 16 & $9-9$ & No \\
\hline 9 & $34+5(243)$ & CS & M & 2180 & $10-50$ & 37 & $8-9$ & No \\
\hline 10 & $34+3(241)$ & CS & $\mathrm{F}$ & 1710 & $10-50$ & 13 & $9-9$ & Yes \\
\hline 11 & $33+0(231)$ & CS & $\mathrm{F}$ & 2115 & $50-90$ & 76 & $7-8$ & Yes \\
\hline 12 & $36+3(255)$ & CS & $\mathrm{F}$ & 2250 & $10-50$ & 17 & $9-9$ & No \\
\hline 13 & $36+6(258)$ & CS & $\mathrm{F}$ & 2340 & $10-50$ & 10 & $9-9$ & No \\
\hline 14 & $32+2(226)$ & CS & $F$ & 1920 & $50-90$ & 79 & $6-8$ & Yes \\
\hline 15 & $32+0(224)$ & CS & $F$ & 1550 & $10-50$ & 31 & $8-8$ & Yes \\
\hline 16 & $34+1$ (239) & Vaginal & $\mathrm{F}$ & 2350 & $50-90$ & 93 & $7-8$ & Yes \\
\hline 17 & $37+4(263)$ & Vaginal & $F$ & 2820 & $10-50$ & 29 & $9-9$ & No \\
\hline 18 & $31+6(223)$ & CS & M & 1365 & $10-50$ & 19 & $8-8$ & Yes \\
\hline 19 & $38+3(269)$ & Vaginal & $\mathrm{F}$ & 3180 & $50-90$ & 62 & $9-9$ & No \\
\hline 20 & $35+5(250)$ & $\mathrm{CS}$ & M & 1790 & $<5$ & 2 & $9-9$ & Yes \\
\hline 21 & $36+1(253)$ & Vaginal & $\mathrm{F}$ & 2140 & $5-10$ & 11 & $9-9$ & No \\
\hline 22 & $38+6(272)$ & Vaginal & $F$ & 2760 & $10-50$ & 12 & $9-9$ & No \\
\hline 23 & $38+5(271)$ & Vaginal & F & 3000 & $10-50$ & 29 & $9-9$ & No \\
\hline 24 & $36+6(258)$ & Vaginal & $F$ & 2600 & $10-50$ & 29 & $8-8$ & No \\
\hline 25 & $36+5(257)$ & Vaginal & $\mathrm{F}$ & 2740 & $10-50$ & 44 & $9-9$ & No \\
\hline 26 & $37+2(261)$ & Vaginal & M & 2580 & $10-50$ & 18 & $8-9$ & No \\
\hline 27 & $31+6(223)$ & $\mathrm{CS}$ & $F$ & 1670 & $10-50$ & 56 & $8-8$ & Yes \\
\hline 28 & $37+1(260)$ & Vaginal & M & 3070 & $10-50$ & 55 & $9-9$ & No \\
\hline 29 & $36+6(258)$ & Vaginal & $F$ & 2830 & $10-50$ & 50 & $9-9$ & No \\
\hline 30 & $36+1(253)$ & Vaginal & $F$ & 2250 & $10-50$ & 22 & $9-9$ & No \\
\hline 31 & $35+6(251)$ & CS & $F$ & 2020 & $10-50$ & 23 & $9 / 9$ & No \\
\hline \multirow[t]{2}{*}{32 (twin) } & \multirow[t]{2}{*}{$31+4(221)$} & \multirow[t]{2}{*}{ CS } & ${ }^{\mathrm{a}} \mathrm{M}$ & 1270 & $5-10$ & 16 & $4-7$ & Yes \\
\hline & & & $F$ & 1275 & $10-50$ & 22 & $7-8$ & Yes \\
\hline \multirow[t]{2}{*}{33 (twin) } & \multirow[t]{2}{*}{$36+4(256)$} & \multirow[t]{2}{*}{$\mathrm{CS}$} & $\mathrm{F}$ & 2350 & $10-50$ & 16 & $9-9$ & No \\
\hline & & & M & 2400 & $10-50$ & 12 & $8-9$ & No \\
\hline \multirow[t]{2}{*}{34 (twin) } & \multirow[t]{2}{*}{$35+6(251)$} & \multirow[t]{2}{*}{ CS } & M & 2920 & $50-90$ & 72 & $8 / 9$ & No \\
\hline & & & M & 3040 & $50-90$ & 81 & $8 / 9$ & No \\
\hline \multirow[t]{2}{*}{$\begin{array}{l}\text { Summary data: } \\
\text { singletons }\end{array}$} & \multirow{2}{*}{$\begin{array}{l}\text { Below 37w: } 24 \\
\text { (77.4 \%) } \\
\text { Below 34w: } 10 \\
(32.3 \%) \\
\text { Below 28: } 0 \\
\text { Median 35 } \\
(28-38)\end{array}$} & $\begin{array}{l}\text { CS } \\
17 \\
(54.8 \%)\end{array}$ & $\begin{array}{l}M \\
9(29.0 \%)\end{array}$ & \multirow{2}{*}{$\begin{array}{l}\text { Below } 1500 \mathrm{~g}: 3 \\
(9.7 \%) \\
\text { Below } 2500 \mathrm{~g}: 21 \\
(67.7 \%) \\
\text { Median } 2140 \\
(935-3180)\end{array}$} & $\begin{array}{l}\text { Below 5th: } 3 / 31 \\
(9.7 \%)\end{array}$ & $\begin{array}{l}\text { Below 5th: } 2 / 31 \\
(6.5 \%)\end{array}$ & \multirow{2}{*}{$\begin{array}{l}5 \text { min: } \\
9(6-9) \\
10 \min \\
9(8-9)\end{array}$} & $\begin{array}{l}\text { Yes } \\
14 \\
(45.2 \%)\end{array}$ \\
\hline & & $\begin{array}{l}\text { Vaginal } \\
14 \\
(45.2 \%)\end{array}$ & $\begin{array}{l}F \\
22 \\
(71.0 \%)\end{array}$ & & $\begin{array}{l}\text { Below 10th } \\
4 / 31(12.9 \%)\end{array}$ & $\begin{array}{l}\text { Below 10th } 3 / 31 \\
(9.7 \%) \\
\text { median } \\
29(2-93)\end{array}$ & & $\begin{array}{l}\text { No } \\
17 \\
(54.8 \%)\end{array}$ \\
\hline
\end{tabular}


Table 6 Main Maternal-foetal outcomes, and intrauterine growth: "controls": 42 singleton deliveries

\begin{tabular}{|c|c|c|c|c|c|c|c|c|}
\hline Case & Gestational age & $\begin{array}{l}\text { Type of } \\
\text { delivery }\end{array}$ & $\begin{array}{l}\text { Sex of the } \\
\text { baby }\end{array}$ & Weight (g) & Centile (Parazzini) & Centile (INeS) & $\begin{array}{l}\text { Apgar (1- } \\
5 \text { min) }\end{array}$ & $\mathrm{NICU}$ \\
\hline 1 & $32+5$ & CS & $M$ & 1470 & $10-50$ & 15 & $6-7$ & Yes \\
\hline 2 & $31+6$ & CS & $\mathrm{F}$ & 1500 & $10-50$ & 41 & $7-9$ & Yes \\
\hline 3 & $27+3$ & CS & $F^{a}$ & 700 & / & 16 & $7-7$ & Yes \\
\hline 4 & $29+3$ & CS & M & 610 & $<5$ & 1 & $4-8$ & Yes \\
\hline 5 & $40+3$ & CS & $\mathrm{F}$ & 2750 & $10-50$ & 7 & $8-10$ & No \\
\hline 6 & $36+2$ & CS & M & 3230 & $50-90$ & 86 & $5-7$ & Yes \\
\hline 7 & $37+1$ & CS & M & 2340 & $<5$ & 8 & $9-10$ & No \\
\hline 8 & $33+0$ & CS & $\mathrm{F}$ & 1950 & $10-50$ & 59 & $8-9$ & Yes \\
\hline 9 & $37+0$ & CS & M & 2300 & $<5$ & 5 & $9-10$ & No \\
\hline 10 & $33+5$ & CS & M & 1900 & $10-50$ & 34 & $9-9$ & Yes \\
\hline 11 & $32+1$ & CS & M & 2180 & $50-90$ & 93 & na & Yes \\
\hline 12 & $37+4$ & CS & M & 2870 & $10-50$ & 27 & $10-10$ & No \\
\hline 13 & $36+4$ & CS & $\mathrm{F}$ & 2630 & $10-50$ & 37 & $9-10$ & No \\
\hline 14 & $36+3$ & CS & M & 2650 & $10-50$ & 32 & 8-9 & Yes \\
\hline 15 & $35+4$ & CS & $\mathrm{F}$ & 2400 & $10-50$ & 48 & $10-10$ & No \\
\hline 16 & $37+1$ & CS & $M$ & 2970 & $10-50$ & 45 & $8-10$ & No \\
\hline 17 & $32+0$ & CS & M & 1950 & 50-90 & 81 & $8-8$ & Yes \\
\hline 18 & $34+6$ & CS & $M$ & 2330 & $10-50$ & 49 & $8-10$ & No \\
\hline 19 & $28+4$ & CS & F & 820 & $10-50$ & 17 & $7-9$ & Yes \\
\hline 20 & $25+2$ & CS & $M^{a}$ & 500 & / & 7 & $3-5$ & Yes \\
\hline 21 & $35+4$ & vaginal & $\mathrm{F}$ & 2450 & $10-50$ & 58 & $8-9$ & No \\
\hline 22 & $36+0$ & vaginal & $\mathrm{F}$ & 2600 & $5-10$ & 13 & $9-10$ & No \\
\hline \multirow[t]{2}{*}{$\begin{array}{l}\text { Summary data: } \\
\text { Cagliari }\end{array}$} & \multirow{2}{*}{$\begin{array}{l}\text { Below 37w: } 17(77.3 \%) \\
\text { Below 34w: } 10(45.5 \%) \\
\text { Below 28w: } 2(9.1 \%) \\
\text { median } \\
34.5 \text { (25-40) }\end{array}$} & $\begin{array}{l}\text { CS } \\
20(90.9 \%)\end{array}$ & $\begin{array}{l}M \\
13(59.1 \%)\end{array}$ & \multirow{2}{*}{$\begin{array}{l}\text { Below } 1500 \mathrm{~g}: \\
5(22.7 \%) \\
\text { Below } 2500 \mathrm{~g}: \\
15(68.2 \%)\end{array}$} & $\begin{array}{l}\text { Below } 5 \text { th or below } 28 \text { w: 5/22 } \\
(22.7 \%)\end{array}$ & Below 5th: 1/22 (4.5 \%) & \multirow{2}{*}{$\begin{array}{l}5 \text { min: } \\
8(3-10) \\
10 \min \\
9(5-10)\end{array}$} & $\begin{array}{l}\text { Yes } \\
12 \\
(54.4 \%)\end{array}$ \\
\hline & & $\begin{array}{l}\text { Vaginal } \\
2(9.1 \%)\end{array}$ & $\begin{array}{l}F \\
9(40.9 \%)\end{array}$ & & $\begin{array}{l}\text { Below 10th or below } 28 \mathrm{w} \text { : } 6 / 22 \\
(27.3 \%)\end{array}$ & $\begin{array}{l}\text { Below 10th: 5/22 } \\
(22.7 \%) \\
\text { median } \\
33(1-93)\end{array}$ & & $\begin{array}{l}\text { No } \\
10 \\
(45.5 \%)\end{array}$ \\
\hline 1 & $37+0$ & CS & $\mathrm{F}$ & 3330 & 50-90 & 92 & $9-9$ & No \\
\hline 2 & $31+0$ & CS & M & 1100 & 5-10 & 10 & $9-9$ & Yes \\
\hline 3 & $33+0$ & CS & M & 1425 & $5-10$ & 9 & $7-9$ & Yes \\
\hline 4 & $36+5$ & Vaginal & $\mathrm{F}$ & 2410 & $10-50$ & 24 & $9-9$ & No \\
\hline 5 & $36+2$ & Vaginal & $\mathrm{F}$ & 2160 & $5-10$ & 14 & $9-9$ & No \\
\hline
\end{tabular}


Table 6 Main Maternal-foetal outcomes, and intrauterine growth: "controls": 42 singleton deliveries (Continued)

\begin{tabular}{|c|c|c|c|c|c|c|c|c|}
\hline 6 & $36+5$ & Vaginal & $F$ & 2600 & $10-50$ & 40 & $9-9$ & No \\
\hline 7 & $28+2$ & CS & M & 750 & $5-10$ & 9 & $5-8$ & Yes \\
\hline 8 & $36+2$ & CS & M & 2500 & $10-50$ & 30 & $9-9$ & No \\
\hline 9 & $32+5$ & CS & M & 1300 & 5-10 & 5 & $9-9$ & Yes \\
\hline 10 & $38+0$ & Vaginal & M & 2280 & $<5$ & 2 & $8-8$ & No \\
\hline 11 & $34+2$ & Vaginal & F & 2160 & $10-50$ & 39 & $8-9$ & No \\
\hline 12 & $38+3$ & Vaginal & $\mathrm{F}$ & 3170 & 50-90 & 61 & $9-9$ & No \\
\hline 13 & $37+6$ & Vaginal & $\mathrm{F}$ & 3050 & $50-90$ & 59 & $8-8$ & No \\
\hline 14 & $38+0$ & CS & $M$ & 2565 & 5-10 & 6 & $9-9$ & No \\
\hline 15 & $32+2$ & CS & M & 1440 & $10-50$ & 19 & $7-9$ & Yes \\
\hline 16 & $38+4$ & Vaginal & M & 2850 & $10-50$ & 18 & $7-8$ & No \\
\hline 17 & $35+6$ & Vaginal & $\mathrm{F}$ & 2900 & $50-90$ & 85 & $9-9$ & No \\
\hline 18 & $35+4$ & CS & M & 1620 & $<5$ & 1 & $9-9$ & Yes \\
\hline 19 & $36+6$ & CS & $\mathrm{F}$ & 2510 & $10-50$ & 29 & $9-9$ & No \\
\hline 20 & $37+6$ & CS & M & 3180 & $50-90$ & 59 & $9-9$ & No \\
\hline \multirow[t]{2}{*}{$\begin{array}{l}\text { Summary data: } \\
\text { Torino }\end{array}$} & \multirow{2}{*}{$\begin{array}{l}\text { Below } 37 \text { w: } 13 \text { (65.0\%) } \\
\text { Below } 34 \text { w: } 5 \text { ( } 25.0 \%) \\
\text { Below } 28 \text { w: } 0 \text { median } \\
36 \text { (28-38) }\end{array}$} & $\begin{array}{l}\text { CS } \\
11(55.0 \%)\end{array}$ & $\begin{array}{l}M \\
11(55.0 \%)\end{array}$ & \multirow{2}{*}{$\begin{array}{l}\text { Below } 1500 \text { g: } 5 \text { ( } 25.0 \%) \\
\text { Below } 2500 \text { g: } 10 \\
(50.0 \%) \\
\text { median } \\
2455 \\
(750-3330)\end{array}$} & $\begin{array}{l}\text { Below 5th: } 2 / 20 \\
(10.0 \%)\end{array}$ & $\begin{array}{l}\text { Below 5th: } 2 / 20 \\
(10.0 \%)\end{array}$ & \multirow[t]{2}{*}{$\begin{array}{l}5 \min : 8(5-9) \\
10 \min : 9 \\
(8-9)\end{array}$} & $\begin{array}{l}\text { Yes } \\
6 \\
(30.0 \%)\end{array}$ \\
\hline & & $\begin{array}{l}\text { Vaginal } \\
9(45.0 \%)\end{array}$ & $\begin{array}{l}F \\
9(45.0 \%)\end{array}$ & & $\begin{array}{l}\text { Below 10th 8/20 } \\
(40.0 \%)\end{array}$ & $\begin{array}{l}\text { Below 10th: } 6 / 20 \\
(30.0 \%) \\
\text { median } \\
21.5(1-92)\end{array}$ & & $\begin{array}{l}\text { No } \\
14 \\
(70.0 \%)\end{array}$ \\
\hline Summary data: all & $\begin{array}{l}\text { Below } 37 \text { w: } 30(71.4 \%) \\
\text { Below } 34 \text { w: } 15(35.7 \%) \\
\text { Below } 28 \text { w: } 2(4.8 \%) \\
\text { median } \\
35.5(25-40)\end{array}$ & $\begin{array}{l}\text { CS } \\
31(73.8 \%)\end{array}$ & $\begin{array}{l}M \\
24(57.1 \%)\end{array}$ & $\begin{array}{l}\text { Below } 1500 \mathrm{~g}: 10 \\
(23.8 \%) \\
\text { Below } 2500 \mathrm{~g}: 25 \\
(59.5 \%) \\
\text { median } \\
2335 \\
(500-3330)\end{array}$ & $\begin{array}{l}\text { Below 5th: } \\
7 / 42 \text { (16.7 \%) } \\
\text { Below 10th: 14/42 (33.3 \%) }\end{array}$ & $\begin{array}{l}\text { Below 5th: 3/42 (7.1 \%) } \\
\text { Below 10th: } 11 / 42 \\
(26.2 \%) \\
\text { median } \\
28.0(1-93)\end{array}$ & $\begin{array}{l}5 \text { min: } 8(5-9) \\
10 \min : 9 \\
(8-9)\end{array}$ & $\begin{array}{l}\text { Yes } \\
18 / 42 \\
(42.9 \%)\end{array}$ \\
\hline $\begin{array}{l}\text { P } \\
\text { diet vs controls }\end{array}$ & $\begin{array}{l}\text { Median: } 0.839 \\
\text { (Mann-Whitney) Below 37: } \\
0.759 \\
\text { (Chi2 Yates) } \\
\text { Below 34: } 0.954 \text { (Chi2 Yates) } \\
\text { Below 28: } 0.505 \text { (Fisher) }\end{array}$ & $\begin{array}{l}0.150 \\
\text { Chi2 } \\
\text { (Yates) }\end{array}$ & $\begin{array}{l}0.032 \\
\text { Chi2 } \\
\text { (Yates) }\end{array}$ & $\begin{array}{l}0.742 \\
\text { Mann-Whitney } \\
\text { Below } 1500 \text { g: } 0.104 \\
\text { (Fisher) } \\
\text { Below } 2500 \text { g: } 0.319 \\
\text { (Fisher) }\end{array}$ & $\begin{array}{l}\text { Below 5th: } \\
0.308 \text { (Fisher) } \\
\text { Below 10th: } \\
0.040 \text { (Fisher) }\end{array}$ & $\begin{array}{l}\text { Below 5th: } 0.643 \\
\text { (Fisher) } \\
\text { Below 10th: } \\
0.068 \text { (Fisher) }\end{array}$ & $\begin{array}{l}5 \text { min: } 0.501 \\
10 \text { min: } 0.076 \\
\text { (Mann- } \\
\text { Whitney) }\end{array}$ & $\begin{array}{l}1.000 \\
\text { Chi2 } \\
\text { (Yates) }\end{array}$ \\
\hline
\end{tabular}

Legend: a Neonatal death, CS caesarean section, NICU neonatal Intensive Care Unit, $M$ male, F female, Parazzini Parazzini growth charts, INeS Italian Neonatal Study growth charts. Fisher: one tailed test 


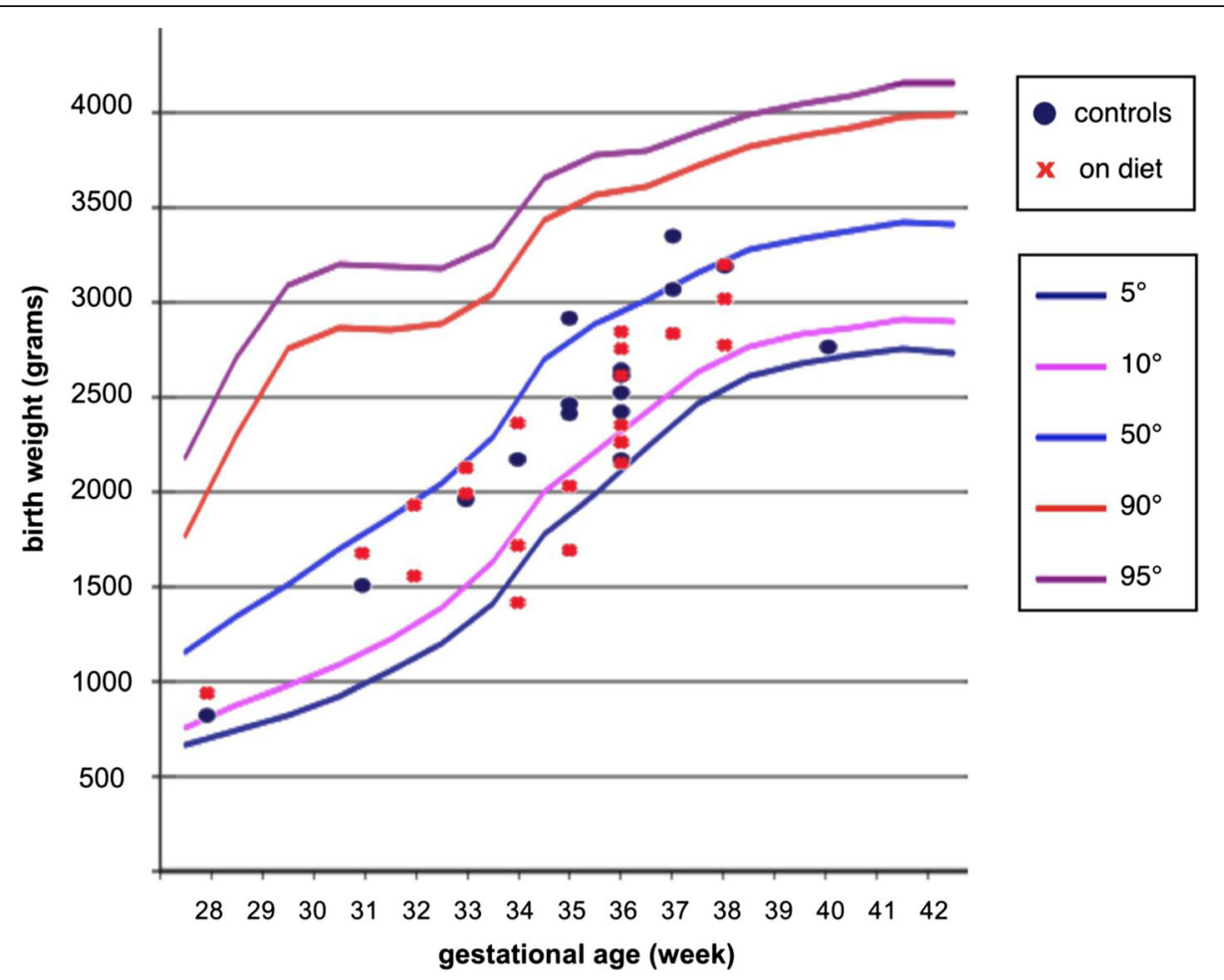

Fig. 4 Relationship between birth-weight and prematurity in on diet patients and controls: females

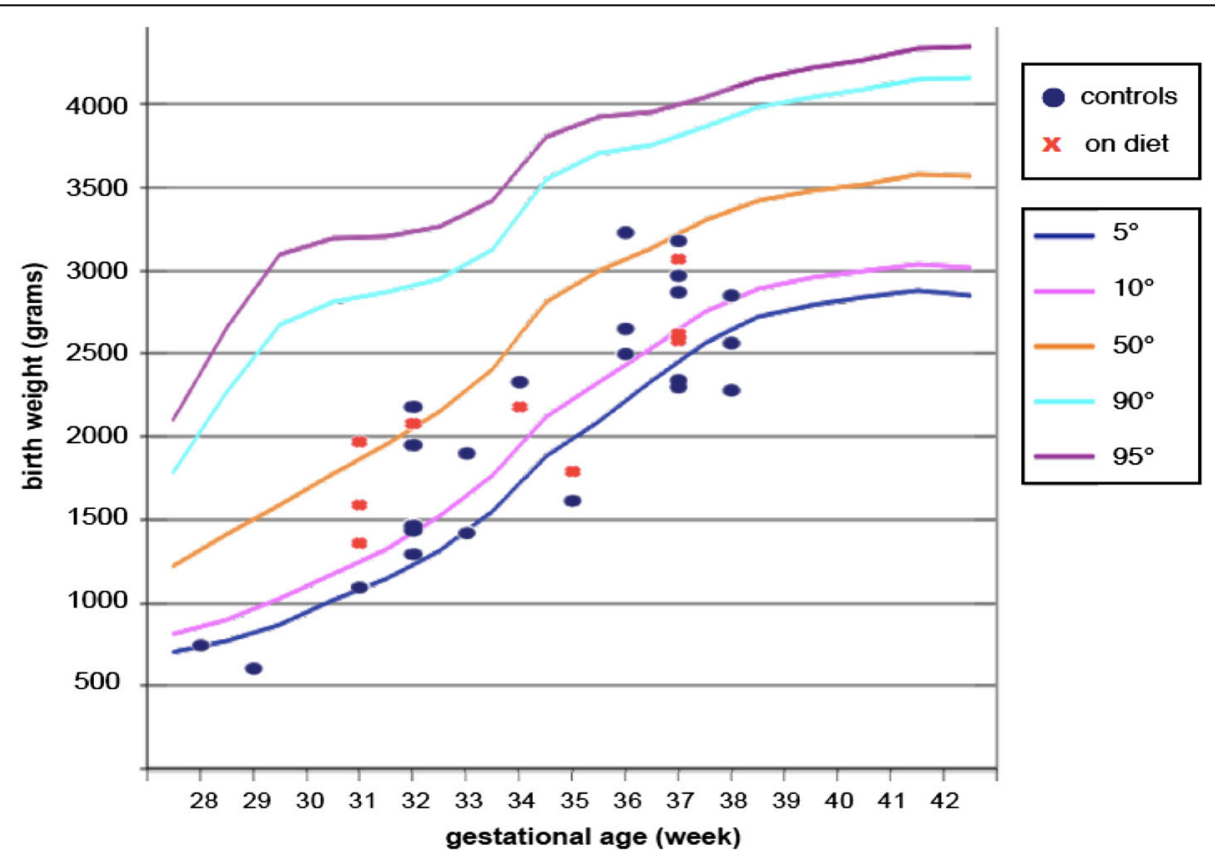

Fig. 5 Relationship between birth-weight and prematurity in on diet patients and controls: males 
the study diet. A growing amount of data suggests that red meat consumption is associated with an increase in cardiovascular risk, while diets that are rich in vegetables, legumes and grains (especially those with a low glycaemic index) may be protective against endothelial dysfunction [53-63].

The specific advantage of vegetable proteins and of supplementation with ketoacids may have played an important role, as it has been suggested in experimental models, which show a protective endothelial effect of ketoacids in rats with kidney disease and a decrease in the risk of CKD in the offspring of rats with genetic kidney diseases that are fed a soya rich diet $[64,65]$.

In the absence of a randomised controlled trial that could present ethical limitations in pregnancy, we hope that our data may stimulate new research on this important issue.

\section{Conclusion}

Vegan-vegetarian diets with moderate protein restriction, supplemented with amino and keto-acids, are safe in pregnancy and may be followed without appreciable side effects. A favourable trend towards improving foetal outcomes was observed for growth and timing of delivery, and reached statistical significance for the combined outcome of small for gestational age babies and extremely preterm delivery, which are also the most robust predictors of future health.

While waiting for further studies to highlight the underlying mechanisms, we hope that this positive finding may raise awareness to the important issue of diet, CKD and pregnancy.

\section{Appendix}

A "vegan/ vegetarian" diet for pregnant women with CKD (moderate protein restriction, supplemented with alphaketo analogues)

This diet is based on some very simple assumptions:

- first point: a low-protein diet is associated with a reduction of the functional "work-load" for the diseased kidneys; this is important since pregnancy increases the "work-load" of the kidneys, which may increase proteinuria and thus reduce kidney function in the long term.

- second point: proteins of vegetable origin cause a lesser "workload" for the kidney as compared to animal-derived proteins, and therefore a "veganvegetarian" diet is better suited to stabilize renal function in pregnant women with chronic kidney disease.

- third point: vegan (no animal or animal-derived food of any kind) and vegetarian (no food from a living source: milk and derivate are allowed) diets are safe in pregnancy (regardless of the presence of
CKD), if well balanced and controlled for protein deficits.

- fourth point: the diet should allow a good quality of life and has to be followed with flexibility, adapting to the preferences of the individual. In pregnancy, we need to pay more attention to maintaining an adequate level of several nutrients, including many vitamins and minerals (the most important ones that we know are: vitamin B12, folic acid, vitamin $\mathrm{D}$, calcium), and to not gaining too much weight (this is why we ask you to pay attention not only to the quality of the food, but also to the quantity).

- fifth point: proteins are contained in "animalderived" food (meat, fish, eggs, poultry and dairy products) and in plant- derived food (grains, cereals, legumes, soya, etc). While some animals (cows for example) are able to build up all the aminoacids from "energy" (i.e., grass), humans are not. Therefore, "animal-derived" proteins are called "noble" or "complete", since they contain ALL the protein components (amino-acids) that we need; plant- derived" proteins are called "incomplete" or "non noble" since their proteins do not contain ALL the aminoacids we need. Every plant-derived protein contains some of them, but we need to put together several different types of plant- derived food to complete them.

- sixth point: the use of "supplements" (called alphakappa or ketosteril that are a mixture of essential aminoacids), allows "completion" of the vegetable proteins, avoiding the risk of nutritional deficits even in patients who do not have time (or like to) combine different "plant-derived" foods.

Important: There are very few studies in the medical literature on low-protein diets in pregnant CKD patients and our experience, obtained in a limited number of patients, is one of the few available ones. To date, the results have been highly positive, also thanks to the close collaboration among us (nephrologists, obstetricians, dieticians) and to the good compliance of the patients that previously followed the diet. Therefore, please report any doubts or side effects immediately to help us follow you better and better understand what we can do to improve our approach.

\section{In short:}

You can eat anything that grows on the earth, under the earth and on trees, and anything that is derived from what grows over and under the earth and on the trees (plants). The only limit is your weight: be careful with fruit it is rich in sugars, and sugar is allowed "with moderation" in pregnancy (PS: olive oil is derived from trees, but has no sugar: it can be used freely). 
You should not eat anything that walks on earth, flies in the air or swims in the water or that is derived from animals, with the exception of butter that contain mainly fat, and should be limited (not too much fat in pregnancy).

However, in the "free" meals you can eat ANYTHING YOU WANT (quality) but NOT TOO MUCH (quantity) to avoid gaining too much weight.

Supplements are prescribed as one tablet every $8-10 \mathrm{~kg}$ of body weight per day, sub-divided over the main meals. The tablets can be taken "in the middle of the meal", at the beginning, or at the end of the meal. The number of pills may increase (or decrease) in pregnancy depending on your condition and biochemical test results.

Supplements are not required in the "free" meals.

\section{In detail}

\section{Breakfast}

For breakfast you can have: tea, coffee, soy drink or soy yoghurt, with bread or biscuits with jam, cereal (such as corn or oat flakes or muesli), or a slice of home made cake (butter, oil, yolk, and a small quantities of milk or yoghurt are allowed) (if you cannot do without, you are allowed to have milk for breakfast...).

Alternatively, for those who prefer a savoury breakfast, you can have bread with olive oil and tomatoes or olives, bread and tofu, crackers or bread sticks with extra virgin olive oil.

\section{1-2 (specify) __ tablets of alpha-kappa}

Lunch and dinner (for each meal), please combine the following

- Pasta or rice or couscous or cereals (like barley, millet, kamut, wheat) seasoned as follows (olive oil is always the best)

- Legumes (for example, chickpeas, peas, beans, lentils, etc)

- Vegetables of any kind (raw or cooked) (see indications for toxoplasmosis)

- Bread or bread sticks or crackers with extra virgin olive oil

- Fresh fruit (indicatively 150-200 g).

2-3 (specify) tablets of alpha-keto analogue

Potatoes can replace bread or pasta.

Legumes should be consumed at least in one main meal in association with pasta, rice or other cereals. If you like, you can use tofu, tempeh or seitan instead of legumes.

Oily nuts such as walnuts (4-6 per day) are useful for their high content of "good" fat (that, like olive oil, protects from atherosclerosis and may help protect the placental vessels).

Snacks

Snacks (mid-morning, mid-afternoon) are welcome:
- 1 cup of soy yoghurt or soy drink

- Bread or biscuits or crackers or bread sticks made with extra virgin olive oil

- Bread with tofu, olives and olive paste, tomato, vegetables

- 1 cup of plain yoghurt or fruit salad

- 1 piece of fresh fruit

- raw vegetables like fennel, celery, peppers, cucumbers, tomatoes, carrots may be used if you are not receptive for toxoplasmosis (please WASH VERY WELL in any case).

- (regular yoghurt may be an alternative, at least occasionally)

\section{Dressings/cooking}

Cook as you prefer (stewed, steamed, grilled, broiled, baked, fried with extra virgin olive oil).

Use extra virgin olive oil for seasoning and avoid mixed seed oil, butter, lard, margarine, cream, sauces (such as mayonnaise, ketchup, tuna sauce, etc.), and items containing sodium glutamate (not good for your vessels or for the placental vessels). Also avoid: vegetable fat, non hydrogenated vegetable fats, palm oil or coconut oil (similar reasons).

Natural spices, herbs (such as rosemary, sage, basil, oregano, thyme, parsley), chili, onion, garlic, lemon juice, vinegar, balsamic vinegar, miso, tamari, shoyu, can be used; however, in case of tamari or miso, please check that they do not have added ingredients.

Use iodized salt, which should not be confused with "sea salt" or "whole salt". Iodized salt is common salt to which iodine has been added (good for thyroid function). In order to increase the intake of some useful minerals (calcium, iron, potassium ...) sesame seeds, sunflower seeds and pumpkin seeds may be added (for example 1 tablespoon).

With regard to baked goods, (crackers, bread sticks), those without added fat or made with extra virgin olive oil, sunflower or corn oil should be preferred.

\section{Drinks and sweets}

Drink water (still or sparkling) throughout the day. Check with your nephrologist to establish the amount. Drink wine and beer only occasionally and in small quantities during pregnancy. Cocktails, spirits and liqueurs are forbidden. Due to the high sugar content, avoid soft drinks, syrups, juices, fruit juices and soluble herbal tea.

Reduce your intake of foods with high sugar content: brown sugar, ice-cream, honey, malt, fruit jellies, croissants, cakes, cream, chocolate, cookies, candy, etc.. Avoid artificial sweeteners like aspartame (E951), acesulfame K (E950), saccharin (E954) and sucralose (E955), cyclamate (E952), neohesperidine DC (E959). 


\section{Toxoplasmosis and listeriosis}

(Two infections that may be transmitted by food). If you are receptive to toxoplasmosis, eat only well-cooked vegetables and meat (during the free meals). CAUTION with STRAWBERRIES, berries, mushrooms, fresh herbs (like parsley, basil, sage ...). In case of doubt, freeze the item to eliminate Toxoplasma. To prevent listeriosis, avoid vacuum-packed products (such as smoked salmon), raw milk, and unpasteurized cheese (such as gorgonzola, taleggio). Check the details with your dietician.

\section{Abbreviations}

CKD: Chronic kidney disease; IUGR: Intrauterine growth restriction; PE: Preeclampsia; SGA: Small for gestational age; TOCOS: Torino Cagliari Observational Study

\section{Funding}

The study received a funding from the University of Torino; GB Piccoli has received a grant and is consultant from the Fresenius Kabi company.

\section{Availability of data and materials}

All the relevant data gathered in a dedicated database as is available in the tables; furter data is at disposal on demand.

\section{Authors' contributions}

RA: drafted the study, followed the patients. FL: designed the diet, followed the patients. SP, FF, IC, LC, MR and MGA, retrieved the data of the patients; made the tables; followed the patients. $V L$ : retrieved the data of the controls; followed the patients. MG, FM, EP made the bibliografic search; retrieved the control data; made the figures; pariticpated to the analysis of the data. EV and $M B$ analysed the data; PA, AP, GC, TT drafted the study, overviewed the research; GBP: designed the diet, drafted the study, followed the patients. All Authors approved the final manuscript.

\section{Competing interests}

The authors declare that they have no competing interests.

\section{Consent for publication}

All patients signed an informed consent to the anonymous use of their clincal data for research purposes.

\section{Ethics approval and consent to participate}

This is an observational study on current clinical practice; The study was approved by the Ethics committee of the OIRM Sant'Anna ( $n^{\circ}$ pratica 335; $n^{\circ}$ protocollo 11551/c28.2 del 4/3/2011). All patients signed a dedicate informed consent.

\section{Author details}

${ }^{1}$ SS Nephrology, SCDU Urology, AOU San Luigi, Orbassano, Italy. ${ }^{2}$ SS Epidemiology, University of Torino, Torino, Italy. ${ }^{3}$ SSD Clinical Nutrition, Department of Clinical and Biological Sciences, University of Torino, Torino, Italy. ${ }^{4}$ SCD Nephrology, Brotzu Hospital, Cagliari, Italy. ${ }^{5}$ SCDU Nephrology, Department Città della Salute e della Scienza, University of Torino, Torino, Italy. 'SS Nephrology, Department of Clinical and Biological Sciences, University of Torino, Regione Gonzole 10, Orbassano, Torino 10100, Italy. ${ }^{7}$ Nèphrologie, $\mathrm{CH}$ du Mans, Le Mans, France.

Received: 2 February 2016 Accepted: 29 August 2016 Published online: 20 September 2016

\section{References}

1. Piccoli GB, Mezza E, Grassi G, Burdese M, Todros T. A 35 year old woman with diabetic nephropathy who wants a baby: case outcome. BMJ. 2004;329:900.2.

2. Piccoli GB, Attini R, Vasario $E$, et al. Vegetarian supplemented low-protein diets. A safe option for pregnant CKD patients: report of 12 pregnancies in 11 patients. Nephrol Dial Transplant. 2011;26:196-205.
3. Piccoli GB, Fassio F, Attini R, et al. Pregnancy in CKD: whom should we follow and why? Nephrol Dial Transplant. 2012;27 Suppl 3:iii111-8.

4. Piccoli GB, Leone F, Attini R, et al. Association of low-protein supplemented diets with fetal growth in pregnant women with CKD. Clin J Am Soc Nephrol. 2014;9:864-73.

5. Piccoli GB, Cabiddu G, Attini R, et al. Risk of adverse pregnancy outcomes in women with CKD. J Am Soc Nephrol. 2015;26:2011-22.

6. Schmidt JA, Rinaldi S, Ferrari $P$, et al. Metabolic profiles of male meat eaters, fish eaters, vegetarians, and vegans from the EPIC-Oxford cohort. Am J Clin Nutr. 2015;102:1518-26.

7. Chiu YF, Hsu CC, Chiu TH, Lee CY, Liu TT, Tsao CK, Chuang SC, Hsiung CA. Cross-sectional and longitudinal comparisons of metabolic profiles between vegetarian and non-vegetarian subjects: a matched cohort study. Br J Nutr. 2015;114:1313-20.

8. Grosso G, Marventano S, Yang J, Micek A, Pajak A, Scalfi L, Galvano F, Kales $\mathrm{SN}$. A comprehensive meta-analysis on evidence of mediterranean diet and cardiovascular disease: Are individual components equal? Crit Rev Food Sci Nutr. 2015;0. [Epub ahead of print].

9. Rees K, Hartley L, Flowers N, et al. 'Mediterranean' dietary pattern for the primary prevention of cardiovascular disease. Cochrane Database Syst Rev. 2013:8:CD009825. doi:10.1002/14651858.CD009825.pub2.

10. Estruch R, Ros E, Salas-Salvadó J, et al. PREDIMED Study Investigators. Primary prevention of cardiovascular disease with a Mediterranean diet. N Engl J Med. 2013;368:1279-90.

11. Wang $X$, Lin $X$, Ouyang $Y Y$, et al. Red and processed meat consumption and mortality: dose-response meta-analysis of prospective cohort studies. Public Health Nutr. 2015;6:1-13.

12. Larsson SC, Orsini N. Red meat and processed meat consumption and allcause mortality: a meta-analysis. Am J Epidemiol. 2014;179:282-9.

13. Ornish D. Holy Cow! What's good for you is good for our planet: comment on "Red Meat Consumption and Mortality". Arch Intern Med. 2012;172:563-4.

14. Mitch WE, Remuzzi G. Diets for patients with chronic kidney disease, still worth prescribing. J Am Soc Nephrol. 2004;15:234-7.

15. Brenner BM, Lawler EV, Mackenzie HS. The hyperfiltration theory: a paradigm shift in nephrology. Kidney Int. 1996;49:1774-7.

16. Neuringer JR, Brenner BM. Hemodynamic theory of progressive renal disease: a 10-year update in brief review. Am J Kidney Dis. 1993;22:98-104.

17. Barsotti G, Guiducci A, Ciardella F, Giovannetti S. Effects on renal function of a low-nitrogen diet supplemented with essential amino acids and ketoanalogues and of hemodialysis and free protein supply in patients with chronic renal failure. Nephron. 1981;27:113-7.

18. Barsotti G, Morelli E, Cupisti A, Meola M, Dani L, Giovannetti S. A lownitrogen low-phosphorus Vegan diet for patients with chronic renal failure. Nephron. 1996;74:390-4.

19. Craig WJ, Mangels AR. American Dietetic Association. J Am Diet Assoc. 2009;109(7):1266-82.

20. American Dietetic Association, Dietitians of Canada. Position of the American Dietetic Association and Dietitians of Canada: Vegetarian diets. J Am Diet Assoc. 2003;103:748-65.

21. Koletzko $B$, Bauer $C P$, Bung $P$, et al. Nutrition in pregnancy - Practice recommendations of the Network "Healthy Start - Young Family Network". Dtsch Med Wochenschr. 2012;137:1366-72.

22. Piccoli GB, Clari R, Vigotti FN, et al. Vegan-vegetarian diets in pregnancy: danger or panacea? A systematic narrative review. BJOG. 2015;122:623-33.

23. Leitzmann C. Vegetarian diets: what are the advantages? Forum Nutr. 2005;57:147-56

24. Key TJ, Appleby PN, Rosell MS. Health effects of vegetarian and vegan diets. Proc Nutr Soc. 2006;65:35-41.

25. Pawlak R, Parrott SJ, Raj S, Cullum-Dugan D, Lucus D. How prevalent is vitamin B (12) deficiency among vegetarians? Nutr Rev. 2013;71:110-7.

26. Woo KS, Kwok TC, Celermajer DS. Vegan diet, subnormal vitamin B-12 status and cardiovascular health. Nutrients. 2014;6:3259-73.

27. Radnitz C, Beezhold B, DiMatteo J. Investigation of lifestyle choices of individuals following a vegan diet for health and ethical reasons. Appetite. 2015;90:31-6.

28. Tucker KL. Vegetarian diets and bone status. Am J Clin Nutr. 2014;100 Suppl 1:329S-35.

29. Mangels AR. Bone nutrients for vegetarians. Am J Clin Nutr. 2014;100 Suppl 1:469S-75.

30. Savva SC, Kafatos A. Is red meat required for the prevention of iron deficiency among children and adolescents? Curr Pediatr Rev. 2014;10(3):177-83. 
31. Turner-McGrievy G, Harris M. Key elements of plant-based diets associated with reduced risk of metabolic syndrome. Curr Diab Rep. $2014 ; 14: 524$

32. Nguyen $R$, Wilcox A. Terms in reproductive and perinatal epidemiology: 2. Perinatal terms. J Epidemiol Community Health. 2005;59:1019-21.

33. Parazzini F, Cortinovis I, Bortolus R, Soliani A, Fedele L. Weight at birth of singleton live births between the 23rd and 27th week of gestation delivered vaginally or by cesarean section. Acta Paediatr. 1994;83:1206-8.

34. www.inescharts.com, last Accessed 29 Nov 2015

35. Cabiddu G, Castellino S, Gernone G, et al. A best practice position statement on pregnancy in chronic kidney disease: the Italian Study Group on Kidney and Pregnancy. J Nephrol. 2016;29:277-303.

36. Piccoli GB, Cabiddu G, Attini R, et al. Pregnancy in CKD: Questions and answers in a changing panorama. Best Pract Res Clin Obstet Gynaecol. 2015:29:625-42.

37. Piccoli GB, Vigotti FN, Leone F, et al. Low-protein diets in CKD: how can we achieve them? A narrative, pragmatic review. Clin Kidney J. 2015:8:61-70.

38. Piccoli GB, Ferraresi M, Deagostini MC, et al. Vegetarian low-protein diets supplemented with keto analogues: a niche for the few or an option for many? Nephrol Dial Transplant. 2013;28:2295-305.

39. Helal I, Fick-Brosnahan GM, Reed-Gitomer B, Schrier RW. Glomerular hyperfiltration: definitions, mechanisms and clinical implications. Nat Rev Nephrol. 2012:8:293-300.

40. Bosch JP, Saccaggi A, Lauer A, Ronco C, Belledonne M, Glabman S. Renal functional reserve in humans: effect of protein intake on glomerular filtration rate. Am J Med. 1983;75:943-50.

41. Brenner BM, Meyer TW, Hostetter TH. Dietary protein intake and the progressive nature of kidney disease. The role of hemodynamically mediated glomerular injury in the pathogenesis of progressive glomerular sclerosis in aging, renal ablation, and intrinsic renal disease. N Engl J Med. 1982;307:652-9.

42. Lindheimer MD, Davison JM, Katz Al. The kidney and hypertension in pregnancy: twenty exciting years. Semin Nephrol. 2001;21:173-89.

43. Jeyabalan A, Conrad KP. Renal function during normal pregnancy and preeclampsia. Front Biosci. 2007;12:2425-37.

44. Heguilén RM, Liste AA, Bellusci AD, Lapidus AM, Bernasconi AR. Renal response to an acute protein challenge in pregnant women with borderline hypertension. Nephrology (Carlton). 2007;12:254-60.

45. Ronco C, Brendolan A, Bragantini L, et al. Renal functional reserve in pregnancy. Nephrol Dial Transplant. 1988;3:157-61.

46. Baylis C. Glomerular filtration rate in normal and abnormal pregnancies. Semin Nephrol. 1999;19:133-9.

47. Piccoli GB, Attini R, Vigotti FN, et al. Is renal hyperfiltration protective in chronic kidney disease-stage 1 pregnancies? A step forward unravelling the mystery of the effect of stage 1 chronic kidney disease on pregnancy outcomes. Nephrology (Carlton). 2015;20:201-8.

48. Blumfield ML, Collins CE. High-protein diets during pregnancy: healthful or harmful for offspring? Am J Clin Nutr. 2014;100:993-5.

49. Imbasciati $E$, Gregorini $G$, Cabiddu G, et al. Pregnancy in CKD stages 3 to 5: fetal and maternal outcomes. Am J Kidney Dis. 2007;49:753-62.

50. Zhang JJ, Ma XX, Hao L, Liu LJ, Lv JC, Zhang H. A systematic review and meta-analysis of outcomes of pregnancy in CKD and CKD outcomes in pregnancy. Clin J Am Soc Nephrol. 2015;10:1964-78.

51. Feng Z, Minard C, Raghavan R. Pregnancy outcomes in advanced kidney disease. Clin Nephrol. 2015;83:272-8.

52. Cabiddu G, Castellino S, Attini R, et al. Pregnancy outcomes after kidney graft in Italy: a long, evolving story (1978-2012). Abstract of the 52ERA-EDTA Congress, London 2015. Nephrol Dial Transplant. 2015;30 suppl 3:iii359.

53. Garneata L, Mircescu G. Effect of low-protein diet supplemented with keto acids on progression of chronic kidney disease. J Ren Nutr. 2013;23:210-3.

54. Brunori G, Viola BF, Parrinello G, et al. Efficacy and safety of a very-low protein diet when postponing dialysis in the elderly: a prospective randomized multicenter controlled study. Am J Kidney Dis. 2007:49:569-80.

55. Defagó MD, Elorriaga N, Irazola VE, Rubinstein AL. Influence of food patterns on endothelial biomarkers: a systematic review. J Clin Hypertens (Greenwich). 2014;16:907-13.

56. Nettleton JA, Steffen LM, Mayer-Davis EJ, et al. Dietary patterns are associated with biochemical markers of inflammation and endothelial activation in the Multi-Ethnic Study of Atherosclerosis (MESA). Am J Clin Nutr. 2006;83:1369-79.
57. Lopez-Garcia E, Schulze MB, Fung TT, et al. Major dietary patterns are related to plasma concentrations of markers of inflammation and endothelial dysfunction. Am J Clin Nutr. 2004;80:1029-35.

58. Wang Y, Mitch WE. Proteins and renal fibrosis: low-protein diets induce Kruppel-like factor-15, limiting renal fibrosis. Kidney Int. 2011;79:933-4.

59. Messina V. Nutritional and health benefits of dried beans. Am J Clin Nutr. 2014;100 Suppl 1:437S-42

60. Flight I, Clifton P. Cereal grains and legumes in the prevention of coronary heart disease and stroke: a review of the literature. Eur J Clin Nutr. 2006;60:1145-59.

61. van den Broek $M$, Leermakers ET, et al. Maternal dietary patterns during pregnancy and body composition of the child at age $6 \mathrm{y}$ : the Generation $\mathrm{R}$ Study. Am J Clin Nutr. 2015;102:873-80.

62. Gao X, Wu J, Dong Z, Hua C, Hu H, Mei C. A low-protein diet supplemented with ketoacids plays a more protective role against oxidative stress of rat kidney tissue with 5/6 nephrectomy than a low-protein diet alone. Br J Nutr. 2010;103:608-16.

63. Gao X, Huang L, Grosjean F, et al. Low-protein diet supplemented with ketoacids reduces the severity of renal disease in 5/6 nephrectomized rats: a role for KLF15. Kidney Int. 2011;79:987-96.

64. Cahill LE, Peng CY, Bankovic-Calic N, Sankaran D, Ogborn MR, Aukema HM. Dietary soya protein during pregnancy and lactation in rats with hereditary kidney disease attenuates disease progression in offspring. $\mathrm{Br} J$ Nutr. 2007:97:77-84.

65. Bonacasa B, Siow RC, Mann GE. Impact of dietary soy isoflavones in pregnancy on fetal programming of endothelial function in offspring. Microcirculation. 2011;18:270-85.

\section{Submit your next manuscript to BioMed Central and we will help you at every step:}

- We accept pre-submission inquiries

- Our selector tool helps you to find the most relevant journal

- We provide round the clock customer support

- Convenient online submission

- Thorough peer review

- Inclusion in PubMed and all major indexing services

- Maximum visibility for your research

Submit your manuscript at www.biomedcentral.com/submit 